\title{
WELL-BALANCED POSITIVITY PRESERVING CENTRAL-UPWIND SCHEME ON TRIANGULAR GRIDS FOR THE SAINT-VENANT SYSTEM
}

\author{
Steve Bryson $^{1}$, Yekaterina Epshteyn ${ }^{2}$, Alexander Kurganov ${ }^{3}$ \\ and Guergana Petrova ${ }^{4}$
}

\begin{abstract}
We introduce a new second-order central-upwind scheme for the Saint-Venant system of shallow water equations on triangular grids. We prove that the scheme both preserves "lake at rest" steady states and guarantees the positivity of the computed fluid depth. Moreover, it can be applied to models with discontinuous bottom topography and irregular channel widths. We demonstrate these features of the new scheme, as well as its high resolution and robustness in a number of numerical examples.
\end{abstract}

Mathematics Subject Classification. 76M12, 35L65.

Received November 20, 2008. Revised May 3, 2010.

Published online October 11, 2010.

\section{INTRODUCTION}

We consider the two-dimensional (2-D) Saint-Venant system of shallow water equations [8]:

$$
\left\{\begin{array}{l}
h_{t}+(h u)_{x}+(h v)_{y}=0 \\
(h u)_{t}+\left(h u^{2}+\frac{1}{2} g h^{2}\right)_{x}+(h u v)_{y}=-g h B_{x} \\
(h v)_{t}+(h u v)_{x}+\left(h v^{2}+\frac{1}{2} g h^{2}\right)_{y}=-g h B_{y}
\end{array}\right.
$$

where the function $B(x, y)$ represents the bottom elevation, $h$ is the fluid depth above the bottom, $(u, v)^{\mathrm{T}}$ is the velocity vector, and $g$ is the gravitational constant. This system is widely used in many scientific and engineering applications related to modeling of water flows in rivers, lakes and coastal areas. The development of robust and accurate numerical methods for the computation of its solutions is important and challenging

\footnotetext{
Keywords and phrases. Hyperbolic systems of conservation and balance laws, semi-discrete central-upwind schemes, Saint-Venant system of shallow water equations.

1 NASA Ames Research Center, Moffett Field, CA 94035, USA. Stephen.T.Bryson@nasa.gov

2 Department of Mathematical Sciences, Carnegie Mellon University, Pittsburgh, PA 15213, USA. rina10@andrew.cmu.edu

3 Mathematics Department, Tulane University, New Orleans, LA 70118, USA. kurganov@math.tulane.edu

4 Department of Mathematics, Texas A\&M University, College Station, TX 77843, USA. gpetrova@math.tamu.edu
} 
problem that has been extensively investigated in the recent years. One of the difficulties encountered is the fact that system (1.1) admits nonsmooth solutions: shocks, rarefaction waves and, when the bottom topography function $B$ is discontinuous, contact discontinuities. In the latter case, the solution may not be unique, which makes the design of robust numerical methods more challenging even in the one-dimensional (1-D) case (see, e.g., [2] and the references therein).

A good numerical method for (1.1) should have two major properties, which are crucial for its stability:

(i) The method should be well-balanced, that is, it should exactly preserve the "lake at rest" steady-state solutions $h+B \equiv$ const., $u \equiv v \equiv 0$ (lake at rest states). This property diminishes the appearance of unphysical waves of magnitude proportional to the grid size (the so-called "numerical storm"), which are normally present when computing quasi steady-states;

(ii) The method should be positivity preserving, that is, the water depth $h$ should be nonnegative at all times. This property ensures a robust performance of the method on dry $(h=0)$ and almost dry $(h \sim 0)$ states.

In the past decade, a number of well-balanced $[4,5,10,15,16,18,21,25,26,29-32,36,37]$ and positivity preserving $[4,18,21,30]$ schemes for (1.1) have been proposed, but only few of them satisfy both major properties (i) and (ii). Among the methods developed are generalizations of the class of accurate, efficient and robust Godunovtype semi-discrete central-upwind schemes, introduced in [19,20,22-24] as universal Riemann-problem-solver-free methods for general multidimensional hyperbolic systems. More precisely, the central-upwind schemes have been extended to compute the solutions of both the 1-D and 2-D Saint-Venant systems. For example, see [18], where well-balanced and positivity preserving central-upwind schemes have been introduced. However, the schemes presented in [18] do not simultaneously satisfy (i) and (ii) over the entire computational domain. In a recent work [21], a new second-order central-upwind scheme, which is well-balanced and positivity preserving at the same time, has been proposed. The key ideas in the development of this scheme are:

- Replacement of the bottom topography function $B$ with its continuous piecewise linear (or bilinear in the 2-D case) approximation;

- Change of conservative variables from $(h, h u, h v)^{T}$ to $(w:=h+B, h u, h v)^{T}$;

- Special positivity preserving correction of the piecewise linear reconstruction for the water surface $w$;

- Development of a special finite-volume-type quadrature for the discretization of the cell averages of the geometric source term.

In both [18,21], the central-upwind schemes for the 2-D system (1.1) are developed for Cartesian grids. Many real world engineering applications require the use of triangular meshes due to the complicated structure of the computational domains of the problems being investigated. A well-balanced central-upwind scheme on triangular grids has been recently developed in [6], where the presented "triangular" scheme is a (nonconservative) modification of the "triangular" central-upwind scheme from [20] with a special quadrature for the source term average over arbitrary triangular cells. The method in [6] has not been tested on examples with (almost) dry states, but since the method is not positivity preserving, it is expected to fail on such states.

In this paper, we present a new second-order semi-discrete central-upwind scheme for computing the solutions of the system (1.1) on triangular grids. Like the central-upwind scheme from [6], our scheme is well-balanced, but the new quadrature for the discretization of the geometric source, presented in Section 2.2, is much simpler than the one proposed in [6]. In addition, unlike the scheme from [6], the proposed central-upwind scheme is positivity preserving. The latter property is achieved by replacing the (possibly discontinuous) bottom topography function $B$ with its continuous piecewise linear approximation (Sect. 2.1) and adjusting the piecewise linear reconstruction for $w$ according to the piecewise linear approximation of $B$ (Sect. 2.3). This technique is borrowed from [21] and naturally adopted to triangular meshes.

The new central-upwind scheme is derived in Section 2 and its positivity preserving property is proved in Section 2.4. In Section 3, we demonstrate the high resolution and robustness of the new scheme on a variety of numerical examples. 


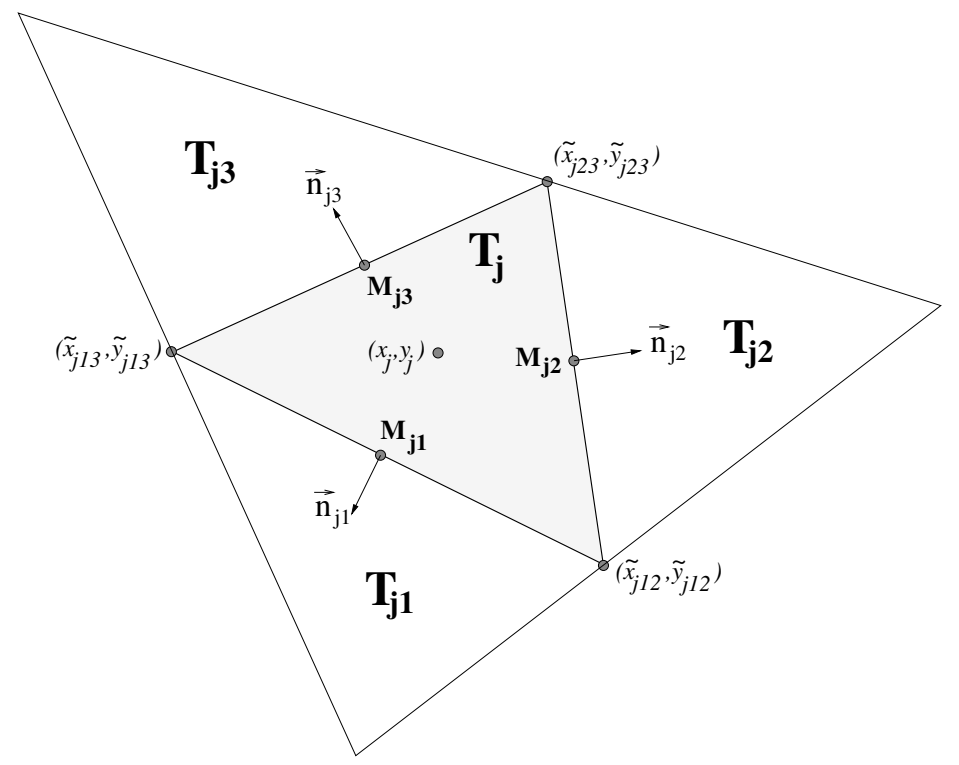

Figure 1. A typical triangular cell with three neighbors.

\section{Description of the SCHEME}

In this section, we describe our new second-order semi-discrete central-upwind scheme for solving the SaintVenant system of shallow water equations on triangular grids. We first denote the water surface by $w:=h+B$ and rewrite (1.1) in terms of the vector $\mathbf{U}:=(w, h u, h v)^{T}$ :

$$
\mathbf{U}_{t}+\mathbf{F}(\mathbf{U}, B)_{x}+\mathbf{G}(\mathbf{U}, B)_{y}=\mathbf{S}(\mathbf{U}, B),
$$

where the fluxes and the source terms are:

$$
\begin{aligned}
& \mathbf{F}(\mathbf{U}, B)=\left(h u, \frac{(h u)^{2}}{w-B}+\frac{1}{2} g(w-B)^{2}, \frac{(h u)(h v)}{w-B}\right)^{T}, \\
& \mathbf{G}(\mathbf{U}, B)=\left(h v, \frac{(h u)(h v)}{w-B}, \frac{(h v)^{2}}{w-B}+\frac{1}{2} g(w-B)^{2}\right)^{T}, \\
& \mathbf{S}(\mathbf{U}, B)=\left(0,-g(w-B) B_{x},-g(w-B) B_{y}\right)^{T} .
\end{aligned}
$$

We assume that a triangulation $\mathcal{T}:=\bigcup_{j} T_{j}$ of the computational domain, consisting of triangular cells $T_{j}$ of size $\left|T_{j}\right|$, is given. We denote by $\vec{n}_{j k}:=\left(\cos \left(\theta_{j k}\right), \sin \left(\theta_{j k}\right)\right)$ the outer unit normals to the corresponding sides of $T_{j}$ of length $\ell_{j k}, k=1,2,3$, see Figure 1 . Let $\left(x_{j}, y_{j}\right)$ be the coordinates of the center of mass for $T_{j}$ and $M_{j k}=\left(x_{j k}, y_{j k}\right)$ be the midpoint of the $k$-th side of the triangle $T_{j}, k=1,2,3$. We denote by $T_{j 1}, T_{j 2}$ and $T_{j 3}$ the neighboring triangles that share a common side with $T_{j}$.

A semi-discrete scheme for (2.1) is a system of ODEs for the approximations of the cell averages of the solution:

$$
\overline{\mathbf{U}}_{j}(t) \approx \frac{1}{\left|T_{j}\right|} \int_{T_{j}} \mathbf{U}(x, y, t) \mathrm{d} x \mathrm{~d} y .
$$


We refer the reader to [20], where a general form of a "triangular" central-upwind scheme for systems of hyperbolic conservation laws is derived. Its second-order version (see $[6,20])$ reads:

$$
\begin{aligned}
\frac{\mathrm{d} \overline{\mathbf{U}}_{j}}{\mathrm{~d} t}= & -\frac{1}{\left|T_{j}\right|} \sum_{k=1}^{3} \frac{\ell_{j k} \cos \left(\theta_{j k}\right)}{a_{j k}^{\text {in }}+a_{j k}^{\text {out }}}\left[a_{j k}^{\text {in }} \mathbf{F}\left(\mathbf{U}_{j k}\left(M_{j k}\right), B\left(M_{j k}\right)\right)+a_{j k}^{\text {out }} \mathbf{F}\left(\mathbf{U}_{j}\left(M_{j k}\right), B\left(M_{j k}\right)\right)\right] \\
& -\frac{1}{\left|T_{j}\right|} \sum_{k=1}^{3} \frac{\ell_{j k} \sin \left(\theta_{j k}\right)}{a_{j k}^{\text {in }}+a_{j k}^{\text {out }}}\left[a_{j k}^{\text {in }} \mathbf{G}\left(\mathbf{U}_{j k}\left(M_{j k}\right), B\left(M_{j k}\right)\right)+a_{j k}^{\text {out }} \mathbf{G}\left(\mathbf{U}_{j}\left(M_{j k}\right), B\left(M_{j k}\right)\right)\right] \\
& +\frac{1}{\left|T_{j}\right|} \sum_{k=1}^{3} \ell_{j k} \frac{a_{j k}^{\text {in }} a_{j k}^{\text {out }}}{a_{j k}^{\text {in }}+a_{j k}^{\text {out }}}\left[\mathbf{U}_{j k}\left(M_{j k}\right)-\mathbf{U}_{j}\left(M_{j k}\right)\right]+\overline{\mathbf{S}}_{j},
\end{aligned}
$$

where $\mathbf{U}_{j}\left(M_{j k}\right)$ and $\mathbf{U}_{j k}\left(M_{j k}\right)$ are the corresponding values at $M_{j k}$ of the piecewise linear reconstruction

$$
\widetilde{\mathbf{U}}(x, y):=\overline{\mathbf{U}}_{j}+\left(\mathbf{U}_{x}\right)_{j}\left(x-x_{j}\right)+\left(\mathbf{U}_{y}\right)_{j}\left(y-y_{j}\right), \quad(x, y) \in T_{j}
$$

of $\mathbf{U}$ at time $t$, that is:

$$
\mathbf{U}_{j}\left(M_{j k}\right):=\lim _{(x, y) \rightarrow M_{j k} ;(x, y) \in T_{j}} \widetilde{\mathbf{U}}(x, y), \quad \mathbf{U}_{j k}\left(M_{j k}\right):=\lim _{(x, y) \rightarrow M_{j k} ;(x, y) \in T_{j k}} \widetilde{\mathbf{U}}(x, y) .
$$

The numerical derivatives $\left(\mathbf{U}_{x}\right)_{j}$ and $\left(\mathbf{U}_{y}\right)_{j}$ are (at least) first-order, componentwise approximations of $\mathbf{U}_{x}\left(x_{j}, y_{j}, t\right)$ and $\mathbf{U}_{y}\left(x_{j}, y_{j}, t\right)$, respectively, computed via a nonlinear limiter, used to minimize the oscillations of the reconstruction (2.6). One can use any nonlinear limiter. A variety of nonoscillatory reconstructions can be found, for example, in $[1,3,7,9,13,14,17,20,35]$. In our numerical experiments, we have used a componentwise piecewise linear reconstruction, which is a modification of the Cartesian grid minmod reconstruction (see, e.g., $[27,28,33,34])$. To calculate the numerical derivatives of the $i$ th component of $\mathbf{U},\left(\mathbf{U}_{x}^{(i)}\right)_{j}$ and $\left(\mathbf{U}_{y}^{(i)}\right)_{j}$, we construct three linear interpolations $L_{j}^{12}(x, y), L_{j}^{23}(x, y)$ and $L_{j}^{13}(x, y)$, which are conservative on the triangle $T_{j}$ and two of the neighboring triangles $\left(T_{j 1}, T_{j 2}\right),\left(T_{j 2}, T_{j 3}\right)$ and $\left(T_{j 1}, T_{j 3}\right)$, respectively. More precisely, the plane $L_{j}^{12}(x, y)$, for example, passes through the points whose first two coordinates are the coordinates of the centers of mass of the triangles $T_{j}, T_{j 1}$ and $T_{j 2}$, and the third coordinates are the cell averages of $\mathbf{U}^{(i)}$ over the corresponding triangles. We then select the linear piece with the smallest magnitude of the gradient, say, $L_{j}^{k m}(x, y)$, and set

$$
\left(\left(\mathbf{U}_{x}^{(i)}\right)_{j},\left(\mathbf{U}_{y}^{(i)}\right)_{j}\right)^{T}=\nabla L_{j}^{k m}
$$

We minimize the oscillations by checking the appearance of local extrema at the points $M_{j k}, 1,2,3$. If for some $k$ one of the reconstructed point values of the $i$ th component of $\mathbf{U}, \mathbf{U}_{j}^{(i)}\left(M_{j k}\right)$ or $\mathbf{U}_{j k}^{(i)}\left(M_{j k}\right)$, is not between the cell averages $\overline{\mathbf{U}}_{j}^{(i)}$ and $\overline{\mathbf{U}}_{j k}^{(i)}$, we replace (2.8) by

$$
\left(\mathbf{U}_{x}^{(i)}\right)_{j}=\left(\mathbf{U}_{y}^{(i)}\right)_{j}=0
$$

The quantity $\overline{\mathbf{S}}_{j}$ in (2.5) is an appropriate discretization of the cell averages of the source term:

$$
\overline{\mathbf{S}}_{j}(t) \approx \frac{1}{\left|T_{j}\right|} \int_{T_{j}} \mathbf{S}(\mathbf{U}(x, y, t), B(x, y)) \mathrm{d} x \mathrm{~d} y .
$$

In Section 2.2, we discuss in detail how to compute $\overline{\mathbf{S}}_{j}$ in a simple way, which guarantees the well-balanced property of the proposed scheme. 
Finally, the directional local speeds $a_{j k}^{\text {in }}$ and $a_{j k}^{\text {out }}$ in (2.5) are defined by

$$
\begin{aligned}
& a_{j k}^{\text {in }}\left(M_{j k}\right)=-\min \left\{\lambda_{1}\left[V_{j k}\left(\mathbf{U}_{j}\left(M_{j k}\right)\right)\right], \lambda_{1}\left[V_{j k}\left(\mathbf{U}_{j k}\left(M_{j k}\right)\right)\right], 0\right\}, \\
& a_{j k}^{\text {out }}\left(M_{j k}\right)=\max \left\{\lambda_{3}\left[V_{j k}\left(\mathbf{U}_{j}\left(M_{j k}\right)\right)\right], \lambda_{3}\left[V_{j k}\left(\mathbf{U}_{j k}\left(M_{j k}\right)\right)\right], 0\right\},
\end{aligned}
$$

where $\lambda_{1}\left[V_{j k}\right] \leq \lambda_{2}\left[V_{j k}\right] \leq \lambda_{3}\left[V_{j k}\right]$ are the eigenvalues of the matrix

$$
V_{j k}=\cos \left(\theta_{j k}\right) \frac{\partial \mathbf{F}}{\partial \mathbf{U}}+\sin \left(\theta_{j k}\right) \frac{\partial \mathbf{G}}{\partial \mathbf{U}},
$$

(the explicit expression of the eigenvalues are given in (2.29) below). If both $a_{j k}^{\text {in }}$ and $a_{j k}^{\text {out }}$ are zero (or very close to zero), the scheme (2.5) reduces to

$$
\begin{aligned}
\frac{\mathrm{d} \overline{\mathbf{U}}_{j}}{\mathrm{~d} t}= & -\frac{1}{\left|T_{j}\right|} \sum_{k=1}^{3} \frac{\ell_{j k} \cos \left(\theta_{j k}\right)}{2}\left[\mathbf{F}\left(\mathbf{U}_{j k}\left(M_{j k}\right), B\left(M_{j k}\right)\right)+\mathbf{F}\left(\mathbf{U}_{j}\left(M_{j k}\right), B\left(M_{j k}\right)\right)\right] \\
& -\frac{1}{\left|T_{j}\right|} \sum_{k=1}^{3} \frac{\ell_{j k} \sin \left(\theta_{j k}\right)}{2}\left[\mathbf{G}\left(\mathbf{U}_{j k}\left(M_{j k}\right), B\left(M_{j k}\right)\right)+\mathbf{G}\left(\mathbf{U}_{j}\left(M_{j k}\right), B\left(M_{j k}\right)\right)\right]+\overline{\mathbf{S}}_{j} .
\end{aligned}
$$

In all reported numerical experiments, we switched from (2.5) to (2.10) when $a_{j k}^{\text {in }}+a_{j k}^{\text {out }}<10^{-8}$.

A fully discrete scheme is obtained from (2.5) (or (2.10)) by using a stable ODE solver of an appropriate order. In our numerical experiments, we have used the third-order SSP-RK ODE solver, see [12]. The time step size should satisfy the CFL condition, see [20]:

$$
\Delta t<\frac{1}{3} \min _{j, k}\left[\frac{r_{j k}}{\max \left\{a_{j k}^{\text {out }}, a_{j k}^{\text {in }}\right\}}\right],
$$

where $r_{j k}, k=1,2,3$ are the three corresponding altitudes of the triangle $T_{j} \in \mathcal{T}$.

\subsection{Piecewise linear approximation of the bottom}

We start by replacing the bottom topography function $B$ with its continuous piecewise linear approximation $\widetilde{B}$, which over each cell $T_{j}$ is given by the formula:

$$
\left|\begin{array}{rrr}
x-\tilde{x}_{j_{12}} & y-\tilde{y}_{j_{12}} & \widetilde{B}(x, y)-\mathcal{B}_{j_{12}} \\
\tilde{x}_{j_{23}}-\tilde{x}_{j_{12}} & \tilde{y}_{j_{23}}-\tilde{y}_{j_{12}} & \mathcal{B}_{j_{23}}-\mathcal{B}_{j_{12}} \\
\tilde{x}_{j_{13}}-\tilde{x}_{j_{12}} & \tilde{y}_{j_{13}}-\tilde{y}_{j_{12}} & \mathcal{B}_{j_{13}}-\mathcal{B}_{j_{12}}
\end{array}\right|=0, \quad(x, y) \in T_{j} .
$$

Here, $\mathcal{B}_{j_{\kappa}}$ are the values of $\widetilde{B}$ at the vertices $\left(\tilde{x}_{j_{\kappa}}, \tilde{y}_{j_{\kappa}}\right), \kappa=12,23,13$, of the cell $T_{j}$ (see Fig. 1), computed according to the following formula:

$$
\mathcal{B}_{j_{\kappa}}:=\frac{1}{2}\left(\max _{\xi^{2}+\eta^{2}=1} \lim _{h, \ell \rightarrow 0} B\left(\tilde{x}_{j_{\kappa}}+h \xi, \tilde{y}_{j_{\kappa}}+\ell \eta\right)+\min _{\xi^{2}+\eta^{2}=1} \lim _{h, \ell \rightarrow 0} B\left(\tilde{x}_{j_{\kappa}}+h \xi, \tilde{y}_{j_{\kappa}}+\ell \eta\right)\right),
$$

which reduces to

$$
\mathcal{B}_{j_{\kappa}}=B\left(\tilde{x}_{j_{\kappa}}, \tilde{y}_{j_{\kappa}}\right)
$$

if the function $B$ is continuous at $\left(\tilde{x}_{j_{\kappa}}, \tilde{y}_{j_{\kappa}}\right)$. Note that when $B$ is discontinuous at this point, formula (2.13) prescribes to $\mathcal{B}_{j_{\kappa}}$ the average of the minimum and maximum values of $B$ at $\left(\tilde{x}_{j_{\kappa}}, \tilde{y}_{j_{\kappa}}\right)$. In any practical case, 
this can be done quite easily. However, if the bottom topography function has a very complicated discontinuous structure, one may use any of the "one-sided" values of $B$ at $\left(\tilde{x}_{j_{\kappa}}, \tilde{y}_{j_{\kappa}}\right)$ as $\mathcal{B}_{j_{\kappa}}$ instead of (2.13).

Let us denote by $B_{j k}$ the value of the continuous piecewise linear reconstruction at $M_{j k}$, namely $B_{j k}:=$ $\widetilde{B}\left(M_{j k}\right)$, and by $B_{j}:=\widetilde{B}\left(x_{j}, y_{j}\right)$ the value of the reconstruction at the center of mass $\left(x_{j}, y_{j}\right)$ of $T_{j}$. Notice that, in general, $B_{j k} \neq B\left(M_{j k}\right)$ and

$$
B_{j}=\frac{1}{\left|T_{j}\right|} \int_{T_{j}} \widetilde{B}(x, y) \mathrm{d} x \mathrm{~d} y
$$

Moreover, one can easily show that

$$
B_{j}=\frac{1}{3}\left(B_{j 1}+B_{j 2}+B_{j 3}\right)=\frac{1}{3}\left(\mathcal{B}_{j_{12}}+\mathcal{B}_{j_{23}}+\mathcal{B}_{j_{13}}\right)
$$

Notice that the approach described above is applicable to any bottom topography function, both continuous and discontinuous.

\subsection{Well-balanced discretization of the source term}

The well-balanced property of the scheme is guaranteed if the discretized cell average of the source term, $\overline{\mathbf{S}}_{j}$, exactly balances the numerical fluxes so that the right-hand side (RHS) of (2.5) vanishes for "lake at rest" steady states $\mathbf{U} \equiv(C, 0,0)^{\mathrm{T}}$, where $C=$ const. Notice that for these states $\mathbf{U}_{j k}\left(M_{j k}\right) \equiv \mathbf{U}_{j}\left(M_{j k}\right) \equiv(C, 0,0)^{\mathrm{T}}, \forall j, k$. After a substitution of a "lake at rest" steady state into (2.5) and taking into account that in this case, $a_{j k}^{\text {in }}=a_{j k}^{\text {out }}$, see (2.9) which becomes (2.29), the source quadrature should satisfy the following two conditions:

$$
-\frac{g}{\left|T_{j}\right|} \sum_{k=1}^{3} \ell_{j k} \cos \left(\theta_{j k}\right) \frac{\left(C-B\left(M_{j k}\right)\right)^{2}}{2}+\overline{\mathbf{S}}_{j}^{(2)}=0
$$

and

$$
-\frac{g}{\left|T_{j}\right|} \sum_{k=1}^{3} \ell_{j k} \sin \left(\theta_{j k}\right) \frac{\left(C-B\left(M_{j k}\right)\right)^{2}}{2}+\overline{\mathbf{S}}_{j}^{(3)}=0
$$

where $\overline{\mathbf{S}}_{j}=\left(0, \overline{\mathbf{S}}_{j}^{(2)}, \overline{\mathbf{S}}_{j}^{(3)}\right)$,

$$
\overline{\mathbf{S}}_{j}^{(2)} \approx-\frac{g}{\left|T_{j}\right|} \int_{T_{j}}(C-B(x, y)) B_{x}(x, y) \mathrm{d} x \mathrm{~d} y
$$

and

$$
\overline{\mathbf{S}}_{j}^{(3)} \approx-\frac{g}{\left|T_{j}\right|} \int_{T_{j}}(C-B(x, y)) B_{y}(x, y) \mathrm{d} x \mathrm{~d} y .
$$

To derive the desired quadrature, we first apply Green's formula,

$$
\int_{T_{j}} \operatorname{div} \overrightarrow{\mathcal{G}} \mathrm{d} x \mathrm{~d} y=\int_{\partial T_{j}} \overrightarrow{\mathcal{G}} \cdot \vec{n} \mathrm{~d} s,
$$


to the vector field $\overrightarrow{\mathcal{G}}=\left(\frac{1}{2}(w(x, y)-B(x, y))^{2}, 0\right)$ and obtain:

$$
\begin{aligned}
-\int_{T_{j}}(w(x, y)-B(x, y)) B_{x}(x, y) \mathrm{d} x \mathrm{~d} y= & \sum_{k=1}^{3} \int_{\left(\partial T_{j}\right)_{k}} \frac{(w(x, y)-B(x, y))^{2}}{2} \cos \left(\theta_{j k}\right) \mathrm{d} s \\
& -\int_{T_{j}}(w(x, y)-B(x, y)) w_{x}(x, y) \mathrm{d} x \mathrm{~d} y,
\end{aligned}
$$

where $\left(\partial T_{j}\right)_{k}$ is the $k$ th side of the triangle $T_{j}, k=1,2,3$. Next, we apply the midpoint rule to the integrals on the RHS of (2.17) and arrive at the following quadrature for the cell average $\overline{\mathbf{S}}_{j}^{(2)}$ :

$$
-\frac{g}{\left|T_{j}\right|} \int_{T_{j}}(w-B) B_{x} \mathrm{~d} x \mathrm{~d} y \approx \frac{g}{2\left|T_{j}\right|} \sum_{k=1}^{3} \ell_{j k}\left(w\left(M_{j k}\right)-B\left(M_{j k}\right)\right)^{2} \cos \left(\theta_{j k}\right)-g w_{x}\left(x_{j}, y_{j}\right)\left(\bar{w}_{j}-\bar{B}_{j}\right),
$$

where

$$
\bar{B}_{j}:=\frac{1}{\left|T_{j}\right|} \int_{T_{j}} B(x, y) \mathrm{d} x \mathrm{~d} y .
$$

Likewise, we obtain the quadrature for the cell average $\overline{\mathbf{S}}_{j}^{(3)}$ :

$$
-\frac{g}{\left|T_{j}\right|} \int_{T_{j}}(w-B) B_{y} \mathrm{~d} x \mathrm{~d} y \approx \frac{g}{2\left|T_{j}\right|} \sum_{k=1}^{3} \ell_{j k}\left(w\left(M_{j k}\right)-B\left(M_{j k}\right)\right)^{2} \sin \left(\theta_{j k}\right)-g w_{y}\left(x_{j}, y_{j}\right)\left(\bar{w}_{j}-\bar{B}_{j}\right) .
$$

Notice that since $w_{x} \equiv w_{y} \equiv 0$ for $w \equiv$ const., the quadratures (2.18)-(2.19) satisfy (2.15)-(2.16) when $\mathbf{U} \equiv(C, 0,0)^{\mathrm{T}}$.

We then replace $B$ with its continuous piecewise linear interpolant $\widetilde{B}$. Since the interpolant $(2.12)$ is second order accurate for smooth $B$, this replacement does not affect the (formal) order of both the central-upwind fluxes in (2.5) and the quadratures (2.18)-(2.19). Finally, the discretization of the source term in (2.5) becomes:

$$
\begin{aligned}
& \overline{\mathbf{S}}_{j}^{(2)}=\frac{g}{2\left|T_{j}\right|} \sum_{k=1}^{3} \ell_{j k}\left(w_{j}\left(M_{j k}\right)-B_{j k}\right)^{2} \cos \left(\theta_{j k}\right)-g\left(w_{x}\right)_{j}\left(\bar{w}_{j}-B_{j}\right), \\
& \overline{\mathbf{S}}_{j}^{(3)}=\frac{g}{2\left|T_{j}\right|} \sum_{k=1}^{3} \ell_{j k}\left(w_{j}\left(M_{j k}\right)-B_{j k}\right)^{2} \sin \left(\theta_{j k}\right)-g\left(w_{y}\right)_{j}\left(\bar{w}_{j}-B_{j}\right),
\end{aligned}
$$

where $B_{j k}=\widetilde{B}\left(M_{j k}\right)$ and $B_{j}$ is given by $(2.14)$.

Remark 2.1. The well-balanced quadrature (2.20) is much simpler than the well-balanced source term discretization proposed in [6].

\subsection{Positivity preserving reconstruction for $\boldsymbol{w}$}

In this section, we describe an algorithm that guarantees positivity of the reconstructed values of the water depth $h_{j}\left(M_{j k}\right), k=1,2,3$, for all $j$, which are obtained from the corresponding point values of $\widetilde{w}$ (obtained with the help of the minmod-type reconstruction, described in the beginning of Sect. 2) and $\widetilde{B}$ :

$$
h_{j}\left(M_{j k}\right):=w_{j}\left(M_{j k}\right)-B_{j k}, \quad k=1,2,3 .
$$


Notice that (see the proof of Thm. 2.2) in order to show that the scheme is positivity preserving, one only needs to verify that $h_{j}\left(M_{j k}\right) \geq 0$. None of the aforementioned second-order piecewise linear (or even the first-order piecewise constant) reconstructions for $w$ can guarantee this since it may obviously happen that the cell average $\bar{w}_{j}>B_{j}$, but $\bar{w}_{j}<B_{j k}$ for some $k$. Therefore, we have to correct the original reconstruction $\widetilde{w}$ so that we ensure the nonnegativity of $h_{j}\left(M_{j k}\right)$ for $k=1,2,3$. There are numerous correction procedures that would guarantee that. The one that we have selected and describe below has less oscillations than some other choices, but we have not conducted a complete study of the dependence of the numerical solution on the the various corrections. Our proposed algorithm modifies $\widetilde{w}$ so that we achieve $\widetilde{w}(x, y) \geq \widetilde{B}(x, y)$ throughout the entire computational domain. The reconstruction $\widetilde{w}$ should be corrected only in those triangles, where $\widetilde{w}\left(\tilde{x}_{j_{\kappa}}, \tilde{y}_{j_{\kappa}}\right)<\mathcal{B}_{j_{\kappa}}$ for some $\kappa$, $\kappa=12,23,13$. Since $\bar{w}_{j} \geq B_{j}$, it is impossible to have $\widetilde{w}\left(\tilde{x}_{j_{\kappa}}, \tilde{y}_{j_{\kappa}}\right)<\mathcal{B}_{j_{\kappa}}$ for all three values of $\kappa$, that is, at all three vertices of the triangle $T_{j}$. Thus, only two cases in which a correction is needed are possible: either there are two indices $\kappa_{1}$ and $\kappa_{2}$, for which $\widetilde{w}\left(\tilde{x}_{j_{\kappa_{1}}}, \tilde{y}_{j_{\kappa_{1}}}\right)<\mathcal{B}_{j_{\kappa_{1}}}$ and $\widetilde{w}\left(\tilde{x}_{j_{\kappa_{2}}}, \tilde{y}_{j_{\kappa_{2}}}\right)<\mathcal{B}_{j_{\kappa_{2}}}$, or there is only one index $\kappa_{1}$, for which $\widetilde{w}\left(\tilde{x}_{j_{\kappa_{1}}}, \tilde{y}_{j_{\kappa_{1}}}\right)<\mathcal{B}_{j_{\kappa_{1}}}$.

In the first case, we will correct the reconstruction $\widetilde{w}$ so that $\widetilde{w}\left(\tilde{x}_{j_{\kappa_{1}}}, \tilde{y}_{j_{\kappa_{1}}}\right)=\mathcal{B}_{j_{\kappa_{1}}}$ and $\widetilde{w}\left(\tilde{x}_{j_{\kappa_{2}}}, \tilde{y}_{j_{\kappa_{2}}}\right)=\mathcal{B}_{j_{\kappa_{2}}}$. These two conditions, together with the conservation requirement for the corrected reconstruction $\widetilde{w}$, uniquely determine the following correction algorithm:

$$
\begin{aligned}
& \text { if } \quad \widetilde{w}\left(\tilde{x}_{{\kappa_{1}}_{1}}, \tilde{y}_{j_{\kappa_{1}}}\right)<\mathcal{B}_{j_{\kappa_{1}}} \text { and } \widetilde{w}\left(\tilde{x}_{j_{\kappa_{2}}}, \tilde{y}_{j_{\kappa_{2}}}\right)<\mathcal{B}_{j_{\kappa_{2}}} \text {, } \\
& \text { set } \widetilde{w}\left(\tilde{x}_{j_{\kappa_{1}}}, \tilde{y}_{j_{\kappa_{1}}}\right):=\mathcal{B}_{j_{\kappa_{1}}} \text { and } \widetilde{w}\left(\tilde{x}_{j_{\kappa_{2}}}, \tilde{y}_{j_{\kappa_{2}}}\right):=\mathcal{B}_{j_{\kappa_{2}}},
\end{aligned}
$$

and replace the linear function, originally reconstructed over the triangle $T_{j}$, with a new function (still denoted by $\widetilde{w}$ ) defined by

$$
\left|\begin{array}{rrr}
x-x_{j} & y-y_{j} & \widetilde{w}(x, y)-\bar{w}_{j} \\
\tilde{x}_{j_{\kappa_{1}}}-x_{j} & \tilde{y}_{j_{\kappa_{1}}}-y_{j} & \mathcal{B}_{j_{\kappa_{1}}}-\bar{w}_{j} \\
\tilde{x}_{j_{\kappa_{2}}}-x_{j} & \tilde{y}_{j_{\kappa_{2}}}-y_{j} & \mathcal{B}_{j_{\kappa_{2}}}-\bar{w}_{j}
\end{array}\right|=0, \quad(x, y) \in T_{j} .
$$

Note that the corrected reconstruction is the restriction over $T_{j}$ of the plane that passes through the three points with coordinates $\left(\tilde{x}_{j_{\kappa_{1}}}, \tilde{y}_{j_{\kappa_{1}}}, \mathcal{B}_{j_{\kappa_{1}}}\right),\left(\tilde{x}_{j_{\kappa_{2}}}, \tilde{y}_{j_{\kappa_{2}}}, \mathcal{B}_{j_{\kappa_{2}}}\right)$, and $\left(x_{j}, y_{j}, \bar{w}_{j}\right)$.

In the second case, we will only need to make sure that after the correction $\widetilde{w}\left(\tilde{x}_{j_{\kappa_{1}}}, \tilde{y}_{j_{\kappa_{1}}}\right)=\mathcal{B}_{j_{\kappa_{1}}}$, while we still have $\widetilde{w}\left(\tilde{x}_{j_{\kappa_{2}}}, \tilde{y}_{j_{\kappa_{2}}}\right) \geq \mathcal{B}_{j_{\kappa_{2}}}$ and $\widetilde{w}\left(\tilde{x}_{j_{\kappa_{3}}}, \tilde{y}_{j_{\kappa_{3}}}\right) \geq \mathcal{B}_{j_{\kappa_{3}}}$. This leaves one degree of freedom in the construction of the corrected linear piece over the triangle $T_{j}$. To minimize the oscillations, we decide to determine $\widetilde{w}\left(\tilde{x}_{j_{\kappa_{2}}}, \tilde{y}_{j_{\kappa_{2}}}\right)$ and $\widetilde{w}\left(\tilde{x}_{j_{\kappa_{3}}}, \tilde{y}_{j_{\kappa_{3}}}\right)$ so that the corresponding distances $\widetilde{w}\left(\tilde{x}_{j_{\kappa_{2}}}, \tilde{y}_{j_{\kappa_{2}}}\right)-\mathcal{B}_{j_{\kappa_{2}}}$ and $\widetilde{w}\left(\tilde{x}_{j_{\kappa_{3}}}, \tilde{y}_{j_{\kappa_{3}}}\right)-\mathcal{B}_{j_{\kappa_{3}}}$ are equal. The conservation requirement and the fact that $\widetilde{w}\left(\tilde{x}_{j_{\kappa_{1}}}, \tilde{y}_{j_{\kappa_{1}}}\right)=\mathcal{B}_{j_{\kappa_{1}}}$ results in the relation

$$
\widetilde{w}\left(\tilde{x}_{j_{\kappa_{2}}}, \tilde{y}_{j_{\kappa_{2}}}\right)-\mathcal{B}_{j_{\kappa_{2}}}=\widetilde{w}\left(\tilde{x}_{j_{\kappa_{3}}}, \tilde{y}_{j_{\kappa_{3}}}\right)-\mathcal{B}_{j_{\kappa_{3}}}=\frac{3}{2}\left(\bar{w}_{j}-B_{j}\right) .
$$

We then replace the original reconstruction over the triangle $T_{j}$, with a new one (still denoted by $\widetilde{w}$ ) defined by

$$
\left|\begin{array}{rrr}
x-x_{j} & y-y_{j} & \widetilde{w}(x, y)-\bar{w}_{j} \\
\tilde{x}_{j_{\kappa_{1}}}-x_{j} & \tilde{y}_{j_{\kappa_{1}}}-y_{j} & \mathcal{B}_{j_{\kappa_{1}}}-\bar{w}_{j} \\
\tilde{x}_{j_{\kappa_{2}}}-x_{j} & \tilde{y}_{j_{\kappa_{2}}}-y_{j} & W-\bar{w}_{j}
\end{array}\right|=0, \quad(x, y) \in T_{j},
$$

where $W=\frac{3}{2}\left(\bar{w}_{j}-B_{j}\right)+\mathcal{B}_{j_{\kappa_{2}}}$. The corrected reconstruction is the restriction over $T_{j}$ of the plane that passes through the three points with coordinates $\left(\tilde{x}_{j_{\kappa_{1}}}, \tilde{y}_{j_{\kappa_{1}}}, \mathcal{B}_{j_{\kappa_{1}}}\right),\left(\tilde{x}_{j_{\kappa_{2}}}, \tilde{y}_{j_{\kappa_{2}}}, W\right)$, and $\left(x_{j}, y_{j}, \bar{w}_{j}\right)$.

The correction procedure (2.22)-(2.25) guarantees that the reconstruction of $w$ is conservative and its values are greater or equal to the corresponding values of $\widetilde{B}$ over the whole triangle $T_{j}$. Hence the point values of the water height, defined by (2.21), will be nonnegative. 
Equipped with the positivity preserving reconstruction $\widetilde{w}$, we now proceed with the computation of the velocities $u$ and $v$, and the one-sided local speeds needed in (2.5). Since the obtained values of $h$ may be very small (or even zero), we calculate the velocities the same way as in [21], namely (we omit the $j, k$ indexes):

$$
u=\frac{\sqrt{2} h(h u)}{\sqrt{h^{4}+\max \left(h^{4}, \varepsilon\right)}}, \quad v=\frac{\sqrt{2} h(h v)}{\sqrt{h^{4}+\max \left(h^{4}, \varepsilon\right)}},
$$

where $\varepsilon$ is a prescribed tolerance (we have taken $\varepsilon=\max _{j}\left\{\left|T_{j}\right|^{2}\right\}$ in all our computations). After evaluating $h$, $u$, and $v$, we recompute the $x$ - and $y$-discharges and fluxes accordingly, that is, we set:

$$
\begin{aligned}
& (h u):=h \cdot u, \quad(h v):=h \cdot v, \\
& \mathbf{F}(\mathbf{U}, B):=\left(h u, h u \cdot u+\frac{1}{2} g(w-B)^{2}, h u \cdot v\right)^{\mathrm{T}}, \\
& \mathbf{G}(\mathbf{U}, B):=\left(h v, h v \cdot u, h v \cdot v+\frac{1}{2} g(w-B)^{2}\right)^{\mathrm{T}},
\end{aligned}
$$

at the points where these quantities are to be calculated. As noted in [21], this is an important step that allows us to preserve the positivity of the fluid depth $h$ (see Thm. 2.2).

Finally, we denote by $u_{j}^{\theta}\left(M_{j k}\right)$ and $u_{j k}^{\theta}\left(M_{j k}\right)$ the normal velocities at the point $M_{j k}$ :

$$
u_{j}^{\theta}\left(M_{j k}\right):=\cos \left(\theta_{j k}\right) u_{j}\left(M_{j k}\right)+\sin \left(\theta_{j k}\right) v_{j}\left(M_{j k}\right), u_{j k}^{\theta}\left(M_{j k}\right):=\cos \left(\theta_{j k}\right) u_{j k}\left(M_{j k}\right)+\sin \left(\theta_{j k}\right) v_{j k}\left(M_{j k}\right),
$$

and write the formulae for the local one-sided speeds of propagation:

$$
\begin{aligned}
& a_{j k}^{\text {out }}=\max \left\{u_{j}^{\theta}\left(M_{j k}\right)+\sqrt{g h_{j}\left(M_{j k}\right)}, u_{j k}^{\theta}\left(M_{j k}\right)+\sqrt{g h_{j k}\left(M_{j k}\right)}, 0\right\}, \\
& a_{j k}^{\text {in }}=-\min \left\{u_{j}^{\theta}\left(M_{j k}\right)-\sqrt{g h_{j}\left(M_{j k}\right)}, u_{j k}^{\theta}\left(M_{j k}\right)-\sqrt{g h_{j k}\left(M_{j k}\right)}, 0\right\} .
\end{aligned}
$$

\subsection{Positivity preserving property of the scheme}

In this section, we prove the positivity preserving property of our new well-balanced central-upwind scheme for triangular grids in the case when the system of ODEs (2.5) is discretized in time, using the forward Euler method or a higher-order SSP ODE solver [12]. The following theorem holds.

Theorem 2.2. Consider the system (2.1)-(2.4) and the central-upwind semi-discrete scheme (2.5)-(2.7), (2.20), (2.22)-(2.25), (2.29). Assume that the system of ODEs (2.5) is solved by the forward Euler method and that for all $j, \bar{w}_{j}^{n}-B_{j} \geq 0$ at time $t=t^{n}$. Then, for all $j, \bar{w}_{j}^{n+1}-B_{j} \geq 0$ at time $t=t^{n+1}=t^{n}+\Delta t$, provided that $\Delta t \leq \frac{1}{6 a} \min _{j, k}\left\{r_{j k}\right\}$, where $a:=\max _{j, k}\left\{a_{j k}^{\text {out }}, a_{j k}^{\text {in }}\right\}$ and $r_{j k}, k=1,2,3$, are the altitudes of triangle $T_{j}$.

Proof. We write the first component in equation (2.5) together with the forward Euler temporal discretization as:

$$
\begin{aligned}
\bar{w}_{j}^{n+1}=\bar{w}_{j}^{n} & -\frac{\Delta t}{\left|T_{j}\right|} \sum_{k=1}^{3} \frac{\ell_{j k} \cos \left(\theta_{j k}\right)}{a_{j k}^{\text {in }}+a_{j k}^{\text {out }}}\left[a_{j k}^{\text {in }}(h u)_{j k}\left(M_{j k}\right)+a_{j k}^{\text {out }}(h u)_{j}\left(M_{j k}\right)\right] \\
& -\frac{\Delta t}{\left|T_{j}\right|} \sum_{k=1}^{3} \frac{\ell_{j k} \sin \left(\theta_{j k}\right)}{a_{j k}^{\text {in }}+a_{j k}^{\text {out }}}\left[a_{j k}^{\text {in }}(h v)_{j k}\left(M_{j k}\right)+a_{j k}^{\text {out }}(h v)_{j}\left(M_{j k}\right)\right] \\
& +\frac{\Delta t}{\left|T_{j}\right|} \sum_{k=1}^{3} \ell_{j k} \frac{a_{j k}^{\text {in }} a_{j k}^{\text {out }}}{a_{j k}^{\text {in }}+a_{j k}^{\text {out }}}\left[w_{j k}\left(M_{j k}\right)-w_{j}\left(M_{j k}\right)\right]
\end{aligned}
$$


where all quantities on the RHS of (2.30) are evaluated at time level $t=t^{n}$. Since the piecewise linear interpolant $\widetilde{B}$ of the bottom topography function is continuous, (2.21) implies that

$$
w_{j k}\left(M_{j k}\right)-w_{j}\left(M_{j k}\right)=h_{j k}\left(M_{j k}\right)-h_{j}\left(M_{j k}\right) .
$$

Moreover, (2.14), (2.21), and the fact that $\bar{w}_{j}^{n}=\frac{1}{3} \sum_{k=1}^{3} w_{j}\left(M_{j k}\right)$ give:

$$
\bar{w}_{j}^{n}-B_{j}=\frac{1}{3} \sum_{k=1}^{3} h_{j}\left(M_{j k}\right)
$$

Using (2.31)-(2.32), subtracting $B_{j}$ from both sides of (2.30), and using the notation (2.28), we arrive at:

$$
\begin{aligned}
\bar{h}_{j}^{n+1}= & \frac{\Delta t}{\left|T_{j}\right|} \sum_{k=1}^{3} h_{j k}\left(M_{j k}\right) \frac{\ell_{j k} a_{j k}^{\text {in }}}{a_{j k}^{\text {in }}+a_{j k}^{\text {out }}}\left[a_{j k}^{\text {out }}-u_{j k}^{\theta}\left(M_{j k}\right)\right] \\
& +\sum_{k=1}^{3} h_{j}\left(M_{j k}\right)\left(\frac{1}{3}-\frac{\Delta t}{\left|T_{j}\right|} \cdot \frac{\ell_{j k} a_{j k}^{\text {out }}}{a_{j k}^{\text {in }}+a_{j k}^{\text {out }}}\left[a_{j k}^{\text {in }}+u_{j}^{\theta}\left(M_{j k}\right)\right]\right),
\end{aligned}
$$

where, as mentioned in (2.27), we have used the fact that $(h u)=h \cdot u$ and $(h v)=h \cdot v$. Next, from the definitions of the local speeds (2.29) we obtain that $a_{j k}^{\text {out }} \geq u_{j k}^{\theta}\left(M_{j k}\right)$ and therefore, all terms in the first sum on the RHS of (2.33) are nonnegative since the corrected reconstruction for $w$ guarantees that $h_{j k}\left(M_{j k}\right) \geq 0$ for all $j$ and $k=1,2,3$. We also obtain:

$$
\frac{\Delta t}{\left|T_{j}\right|} \cdot \frac{\ell_{j k} a_{j k}^{\text {out }}}{a_{j k}^{\text {in }}+a_{j k}^{\text {out }}}\left[a_{j k}^{\text {in }}+u_{j}^{\theta}\left(M_{j k}\right)\right] \leq \frac{\Delta t}{\left|T_{j}\right|} \cdot \frac{\ell_{j k} a_{j k}^{\text {out }}}{a_{j k}^{\text {in }}+a_{j k}^{\text {out }}}\left[a_{j k}^{\text {in }}+a_{j k}^{\text {out }}\right] \leq \frac{1}{3},
$$

provided $\Delta t \leq \frac{1}{3 \alpha} \max _{j, k}\left\{\frac{\left|T_{j}\right|}{\ell_{j k}}\right\}$, where $\alpha:=\max _{j, k}\left\{a_{j k}^{\text {out }}\right\}$. From (2.11) and the fact that $\left|T_{j}\right|=0.5 r_{j k} \ell_{j k}$, we conclude that all terms in the second sum on the RHS of (2.33) are also nonnegative as long as $\Delta t<\frac{1}{6 a} \min _{j k}\left\{r_{j k}\right\}$, $a:=\max _{j, k}\left\{a_{j k}^{\text {out }}, a_{j k}^{\text {in }}\right\}$, since $h_{j}\left(M_{j k}\right) \geq 0$ for all $j$ and $k=1,2,3$. This completes the proof of the theorem.

\section{Remark 2.3.}

1. Notice that Theorem 2.2 is proved under the stricter CFL condition than the one stated in (2.11).

2. Theorem 2.2 is still valid if one uses a higher-order SSP ODE solver (either the Runge-Kutta or the multistep one), because such solvers can be written as a convex combination of several forward Euler steps.

\section{NUMERICAL EXPERIMENTS}

We test our well-balanced positivity preserving central-upwind scheme on several problems in which (almost) dry "lake at rest" steady states and/or their small perturbations are present. These examples clearly demonstrate the ability of the proposed scheme to accurately resolve quasi-steady states (small perturbations of "lake at rest" steady states) and, at the same time, to preserve the positivity of the fluid depth $h$ (as proved in Thm. 2.2).

In all examples that follow, the gravitational constant is $g=1$. In Examples 1-3, the computational domain is a rectangle, and we use the structured triangular mesh outlined in Figure 2, while in Example 4, where we simulate a flow in a converging-diverging channel, the mesh is unstructured, see Figure 16 (right). 


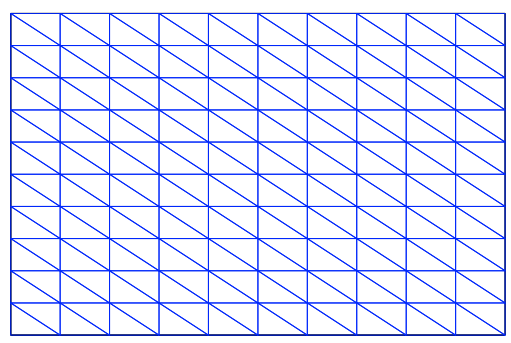

FiguRE 2. Structured triangular mesh. Horizontal to vertical cathetus ratio is 2:1.
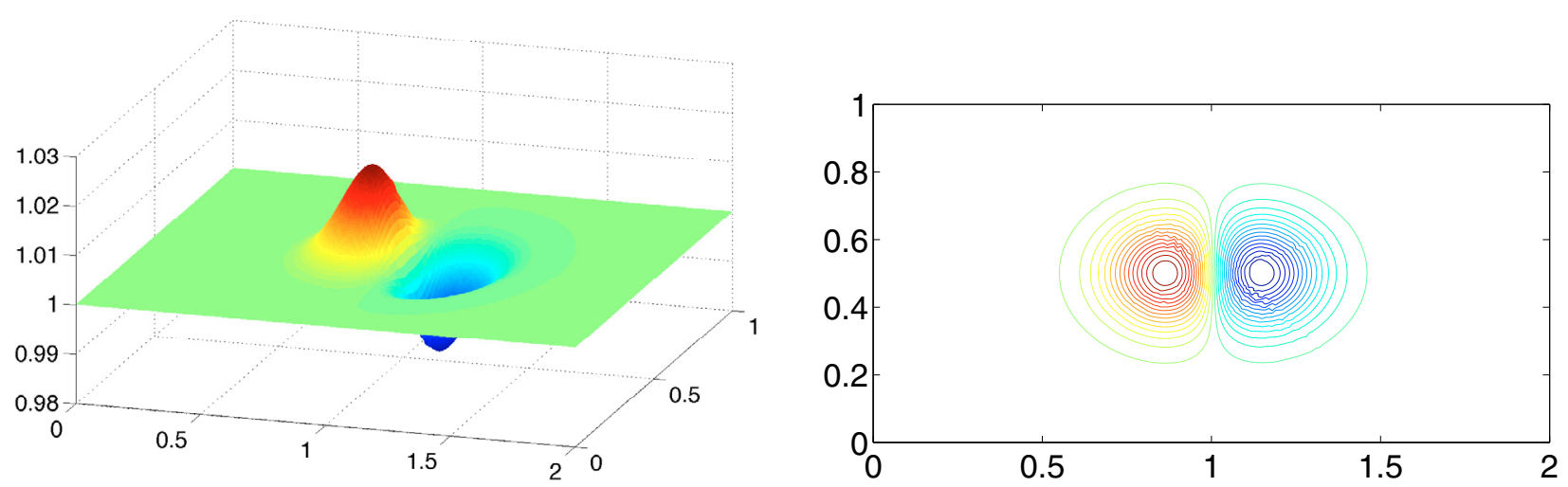

Figure 3. $w$ component of the solution of the IVP $(2.1)-(2.4),(3.1)$ on a $2 \times 400 \times 400$ grid: the 3 -D view (left) and the contour plot (right).

\section{Example 1 - Accuracy test}

The goal of this numerical example is to experimentally check the order of accuracy of the proposed centralupwind scheme. The scheme is applied to the system (2.1)-(2.4) subject to the following initial data and bottom topography:

$$
w(x, y, 0)=1, \quad u(x, y, 0)=0.3, \quad B(x, y)=0.5 \exp \left(-25(x-1)^{2}-50(y-0.5)^{2}\right) .
$$

The computational domain is $[0,2] \times[0,1]$ and we have used a zero-order extrapolation at all boundaries.

For a reference solution, we solve this problem with our method on a grid using $2 \times 400 \times 400$ triangles. By $t=0.07$, the solution converges to the steady state, which is in this case nonconstant but smooth. We show the water surface for the reference solution at time $t=0.07$ in Figure 3 . We use this reference solution to test the numerical convergence. The $L^{1}$ - and $L^{\infty}$-errors are shown in Table 1 . The obtained errors and the rate of convergence are similar to the ones reported in [21], Table 4.1, for the 1-D problem on a uniform grid of the same size. Tests of our method on a finer mesh are prevented by the size of the problem and available computer resources. We believe that further mesh refinement would increase the rates, similar to the behavior shown in [21], Table 4.1.

\section{Example 2 - Small perturbation of a stationary steady-state solution}

Here, we first solve the initial value problem (IVP), which is a modification of the benchmark proposed in [25]. The computational domain is $[0,2] \times[0,1]$ and the bottom consists of an elliptical shaped hump:

$$
B(x, y)=0.8 \exp \left(-5(x-0.9)^{2}-50(y-0.5)^{2}\right) .
$$


TABLE 1. Example 1: $L^{1}$ - and $L^{\infty}$-errors and numerical orders of accuracy.

\begin{tabular}{|c|cc|cc|}
\hline Number of cells & $L^{1}$-error & Order & $L^{\infty}$-error & Order \\
\hline $2 \times 50 \times 50$ & $6.59 \mathrm{e}-04$ & - & $8.02 \mathrm{e}-03$ & - \\
$2 \times 100 \times 100$ & $2.87 \mathrm{e}-04$ & 1.20 & $3.59 \mathrm{e}-03$ & 1.16 \\
$2 \times 200 \times 200$ & $1.00 \mathrm{e}-04$ & 1.52 & $1.21 \mathrm{e}-03$ & 1.57 \\
\hline
\end{tabular}

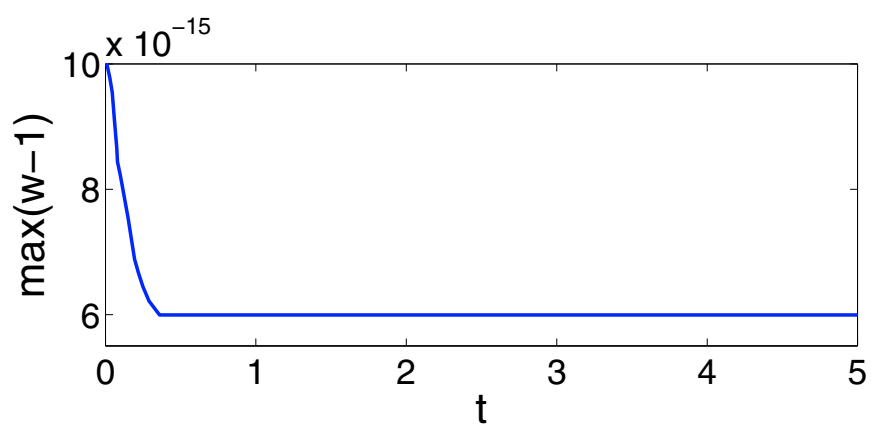

Figure 4. $\max _{x, y}(w-1)$ as a function of $t$, where $w$ is the solution of the IVP (2.1)(2.4), (3.2), (3.3) with $\varepsilon=10^{-14}$.

Initially, the water is at rest and its surface is flat everywhere except for $0.05<x<0.15$ :

$$
w(x, y, 0)=\left\{\begin{array}{lc}
1+\varepsilon, & 0.05<x<0.15, \\
1, & \text { otherwise },
\end{array}(x, y, 0) \equiv v(x, y, 0) \equiv 0,\right.
$$

where $\varepsilon$ is the perturbation height. We have used zero-order extrapolation at the right and the left boundaries, while the boundary conditions in the $y$-direction are periodic.

We first take a very small $\varepsilon=10^{-14}$, which is comparable with the machine error, and numerically verify property (i) of the proposed central-upwind scheme. In Figure 4, we plot $\max _{x, y}(w-1)$ as a function of $t$, computed on a very coarse mesh using $2 \times 10 \times 10$ triangles. As one can clearly see, no instabilities are developed and the balance between the fluxes and the geometric source terms is preserved numerically.

We then take a larger, but still very small perturbation height $\varepsilon=10^{-4}$. Figure 5 (left column) displays the right-going disturbance as it propagates past the hump. The water surface, $w(x, y, t)$, computed on the mesh using $2 \times 200 \times 200$ triangles, is presented at times $t=0.6,0.9,1.2,1.5$ and 1.8. One can observe the high resolution of complex small features of the flow (compare with $[6,18,25]$ ). To demonstrate that the importance of the well-balanced property, we apply a non well-balanced central-upwind scheme, obtained by replacing the quadrature (2.20) with a straightforward midpoint rule discretization:

$$
\overline{\mathbf{S}}_{j}^{(2)}=-g\left(\bar{w}_{j}-B_{j}\right)\left(B_{x}\right)_{j}, \quad \overline{\mathbf{S}}_{j}^{(3)}=-g\left(\bar{w}_{j}-B_{j}\right)\left(B_{y}\right)_{j},
$$

where $\left(B_{x}\right)_{j}$ and $\left(B_{y}\right)_{j}$ are the slopes of the $j$ th piece of the piecewise linear approximation of the bottom topography, $\widetilde{B}$, given by (2.12). A "non well-balanced" solution, computed on the same $2 \times 200 \times 200$ grid, is shown in the right column of Figure 5. As one can clearly see there, the solution, computed by the non wellbalanced scheme contains "parasitic" waves, which are of about the same magnitude as the waves generated by the small perturbation. This clearly demonstrates the advantage of the well-balanced quadrature (2.20). 

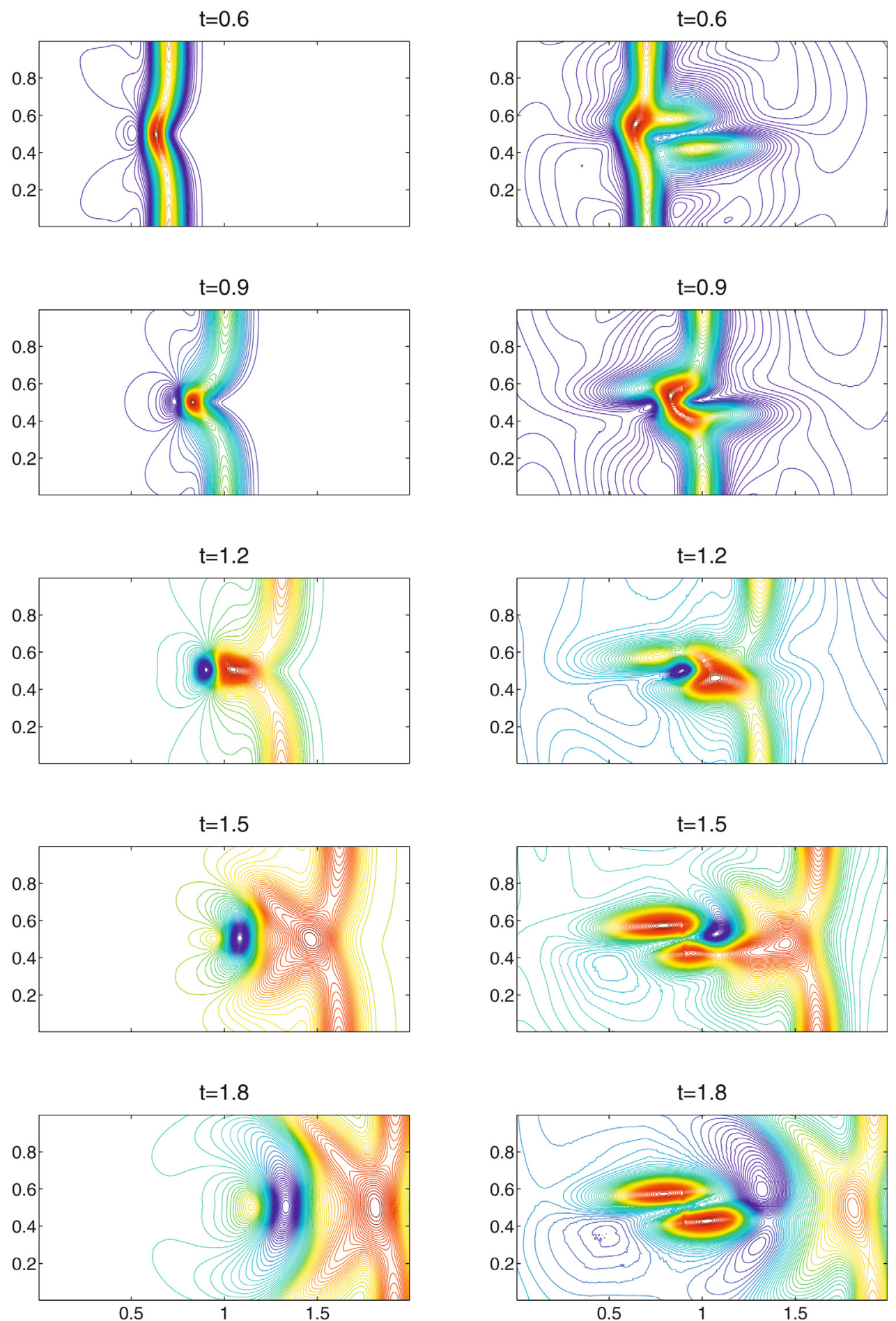

Figure 5. $w$ component of the solution of the IVP (2.1)-(2.4), (3.2), (3.3) with $\varepsilon=10^{-4}$, computed by the well-balanced (left column) and non well-balanced (right column) centralupwind schemes. 

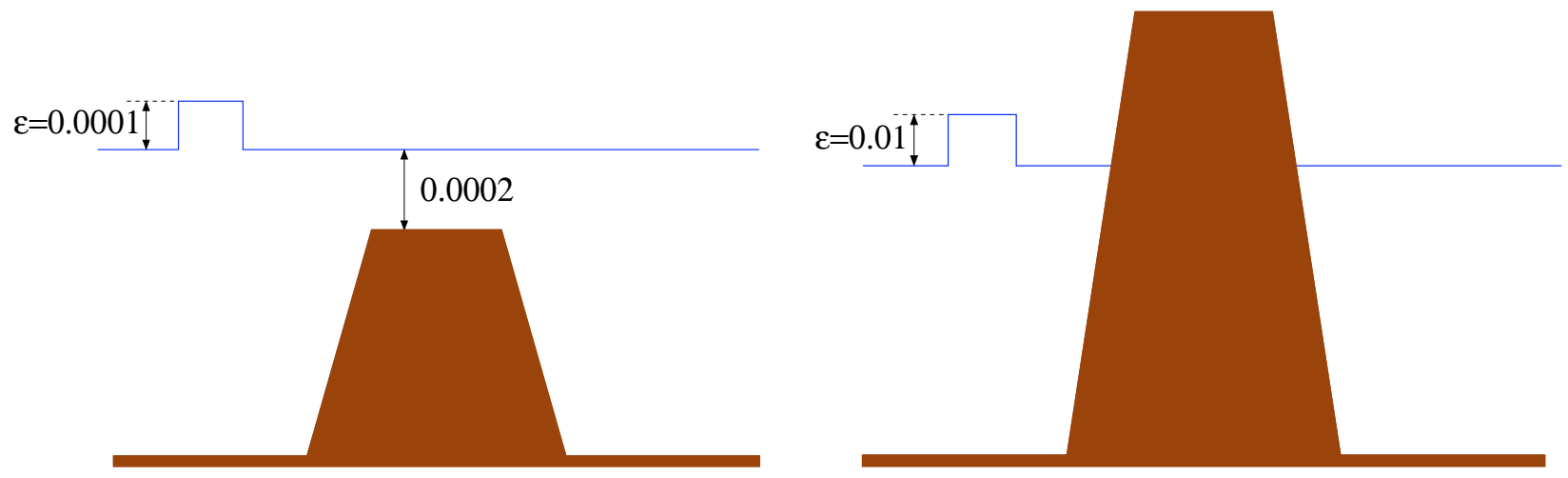

Figure 6. 1-D slice of the bottom topographies (3.4), left, and (3.6), right. These plots are not to scale.
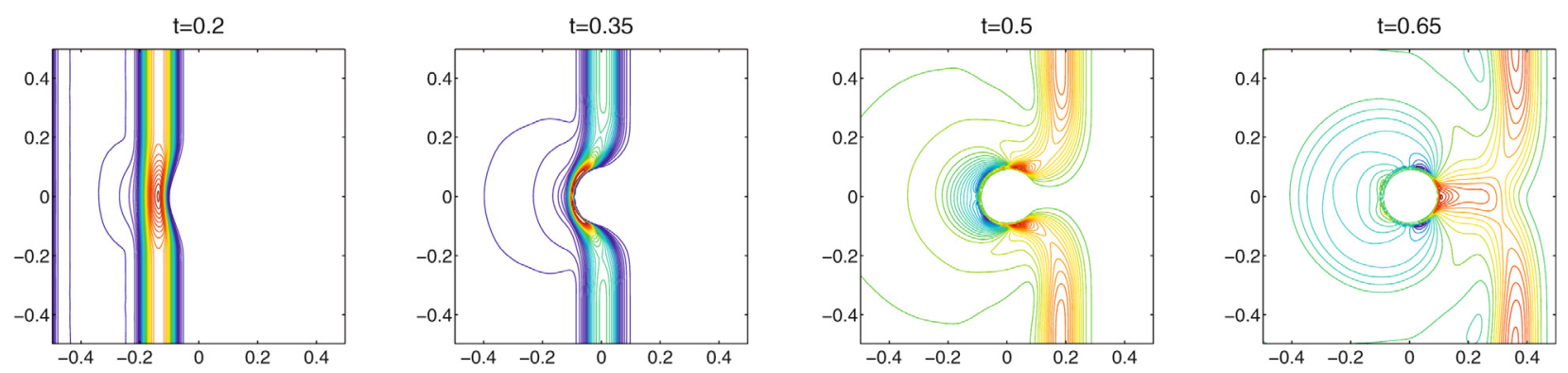

FiguRE 7. $w$ component of the solution of the IVP (2.1)-(2.4), (3.4), (3.5), computed by the well-balanced central-upwind scheme on a $2 \times 200 \times 200$ grid.

Next, we modify the bottom topography (3.2) to numerically study the case of a submerged flat plateau, see Figure 6 (left). Notice that the plateau is very close to the water surface, and that the initial water depth over the plateau is equal to the perturbation height $\varepsilon=10^{-4}$. The computational domain is now $[-0.5,0.5] \times[-0.5,0.5]$, the radially symmetric bottom topography is given by

$$
B(r)=\left\{\begin{array}{lr}
0.9998, & r \leq 0.1 \\
9.998(0.2-r), & 0.1<r<0.2 \\
0, & \text { otherwise }
\end{array}\right.
$$

where $r:=\sqrt{x^{2}+y^{2}}$, and the initial data are:

$$
w(x, y, 0)=\left\{\begin{array}{lc}
1+\varepsilon, & -0.4<x<-0.3, \\
1, & \text { otherwise },
\end{array} \quad u(x, y, 0) \equiv v(x, y, 0) \equiv 0 .\right.
$$

In Figure 7, the solution, computed by the well-balanced central-upwind scheme on the mesh using $2 \times 200 \times$ 200 triangles, is shown at times $t=0.2,0.35,0.5$ and 0.65 . Since the area over the plateau is almost dry, the right-going disturbance mostly bends around that area, while only small portion of the wave propagates over the area. As one can see from Figure 7, the general structure of the solution is well resolved on the $2 \times 200 \times 200$ grid. However, a finer mesh is clearly needed to achieve high resolution of the solution over the plateau area (see Fig. 8, where the solution computed on the mesh using $2 \times 400 \times 400$ triangles is plotted). Notice that the positivity of $h$ is preserved and no instabilities are developed at the almost dry area. 

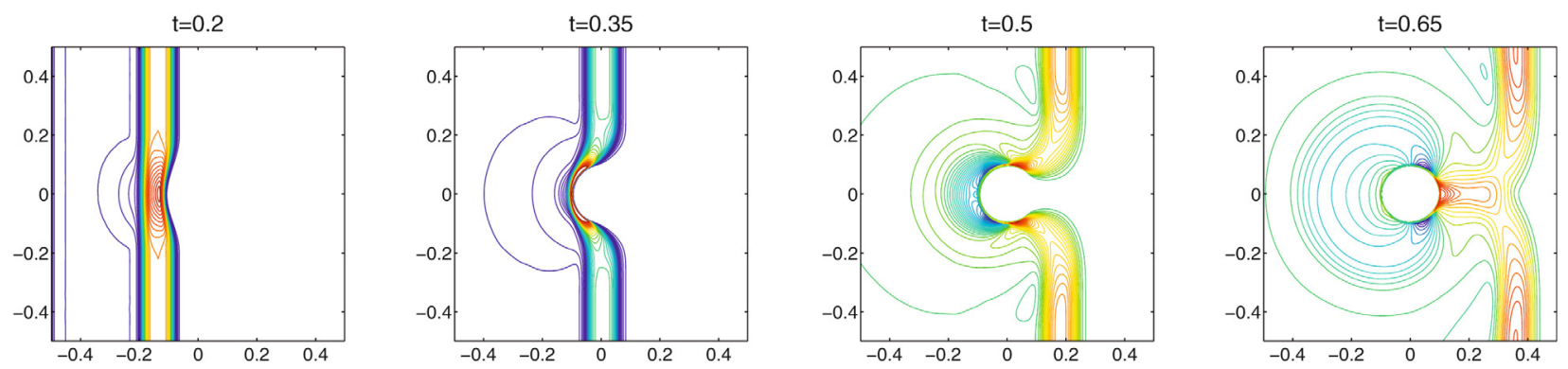

FIGURE 8. $w$ component of the solution of the IVP (2.1)-(2.4), (3.4), (3.5), computed by the well-balanced central-upwind scheme on a $2 \times 400 \times 400$ grid.
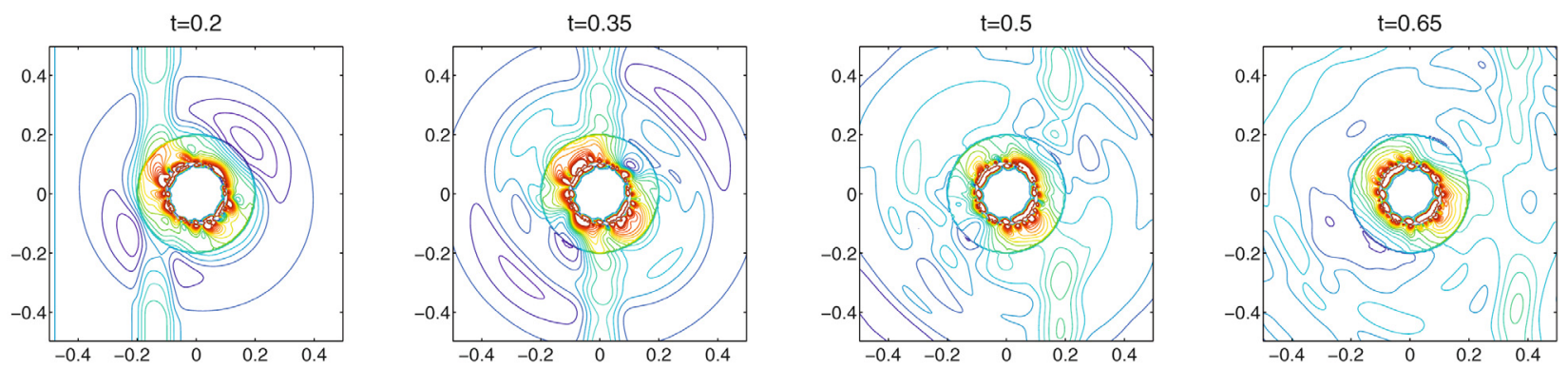

Figure 9. $w$ component of the solution of the IVP (2.1)-(2.4), (3.4), (3.5), computed by a non well-balanced central-upwind scheme on a $2 \times 200 \times 200$ grid (compare with Fig. 7).
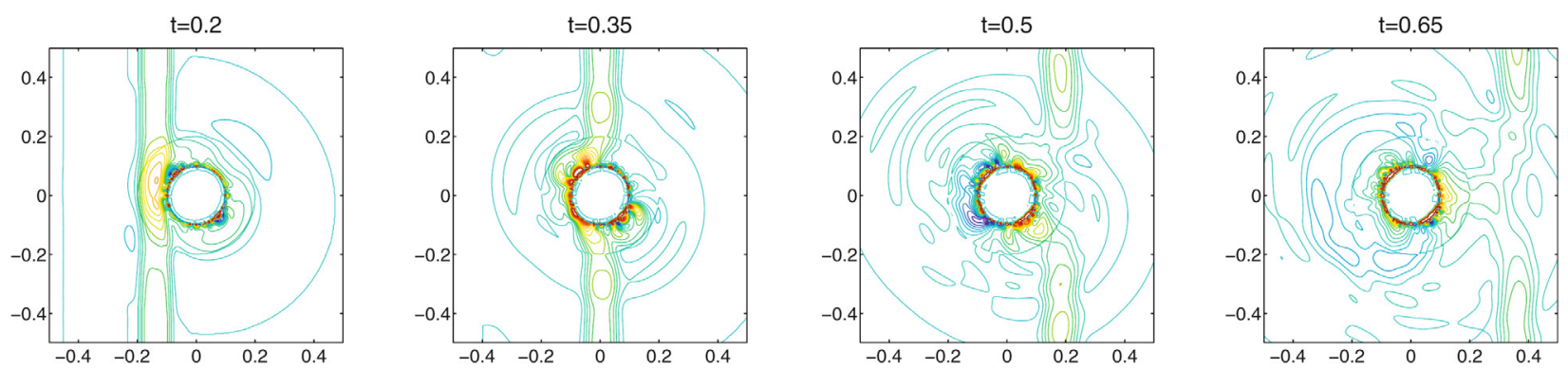

FIGURE 10. $w$ component of the solution of the IVP (2.1)-(2.4), (3.4), (3.5), computed by a non well-balanced central-upwind scheme on a $2 \times 400 \times 400$ grid (compare with Fig. 8).

It is instructive to compare the results, obtained by the well-balanced positivity preserving central-upwind scheme, with the solution, computed by a non well-balanced (yet positivity preserving) one. The latter results are shown in Figures 9 and 10. As one can see, when a coarser $2 \times 200 \times 200$ grid is used (Fig. 9), the "parasitic" waves, generated at the plateau area, are dominating and the computed solution is completely incorrect. When a finer $2 \times 400 \times 400$ mesh is used (Fig. 10), the non well-balanced solution becomes a little less oscillatory, but it is still unsatisfactory. 
In the final part of Example 2, we consider the situation in which the bottom hump is above the water surface so that there is a disk-shaped island at the origin, see Figure 6 (right):

$$
B(r)=\left\{\begin{array}{lr}
1.1, & r \leq 0.1 \\
11(0.2-r), & 0.1<r<0.2 \\
0, & \text { otherwise }
\end{array}\right.
$$

The computational domain is $[-0.5,0.5] \times[-0.5,0.5]$ and the initial data are given by

$$
w(x, y, 0)=\left\{\begin{array}{lc}
1+\varepsilon, & -0.4<x<-0.3, \\
\max \left\{1, B\left(\sqrt{x^{2}+y^{2}}\right)\right\}, & \text { otherwise },
\end{array} u(x, y, 0) \equiv v(x, y, 0) \equiv 0\right.
$$

As one can see from Figure 11, where the computed water surface $w$ is shown, the right-going disturbance bends around the island while the general solution structure is quite similar to the one obtained in the submerged plateau case. The major difference is that in this case $w \neq$ const. at the "lake at rest" steady state and completely dry states and states that change their status back and forth between dry and almost dry (at the areas around the island) are now present. Nevertheless, the solution obtained by the proposed positivity preserving centralupwind scheme remains consistent and stable. This demonstrates the robustness of our method.

\section{Example 3 - Saint-Venant system with friction and discontinuous bottom}

It is a well-known fact that more realistic shallow water models based on the Saint-Venant system (1.1) should include additional friction and/or viscosity terms. In [11], such models were derived from the NavierStokes equations for incompressible flows with a free moving boundary. Presence of friction and viscosity terms guarantees uniqueness of the steady state solution, especially in the case when the fluid propagates into a certain region and gradually occupies parts of initially dry areas, as, for example, in Figure 13.

We consider the simplest model in which only friction terms, $-\kappa(h) u$ and $-\kappa(h) v$, are added to the RHS of the second and third equations in (1.1):

$$
\left\{\begin{array}{l}
h_{t}+(h u)_{x}+(h v)_{y}=0 \\
(h u)_{t}+\left(h u^{2}+\frac{1}{2} g h^{2}\right)_{x}+(h u v)_{y}=-g h B_{x}-\kappa(h) u \\
(h v)_{t}+(h u v)_{x}+\left(h v^{2}+\frac{1}{2} g h^{2}\right)_{y}=-g h B_{y}-\kappa(h) v
\end{array}\right.
$$

We numerically solve the system (3.8) on the domain $[-0.25,1.75] \times[-0.5,0.5]$, assuming that the friction coefficient is $\kappa(h)=0.001(1+10 h)^{-1}$, and the bottom topography function has a discontinuity along the vertical line $x=1$ and is given by:

$$
B(x, y)=100 y^{4}+ \begin{cases}1, & x<0, \\ \cos ^{2}(\pi x), & 0 \leq x \leq 0.4, \\ \cos ^{2}(\pi x)+0.28(\cos (10 \pi(x-0.5))+1), & 0.4 \leq x \leq 0.5, \\ 0.5 \cos ^{4}(\pi x)+0.28(\cos (10 \pi(x-0.5))+1), & 0.5 \leq x \leq 0.6, \\ 0.5 \cos ^{4}(\pi x), & 0.5 \leq x<1, \\ 0.28 \sin (2 \pi(x-1)), & 1<x \leq 1.5, \\ 0, & x>1.5 .\end{cases}
$$



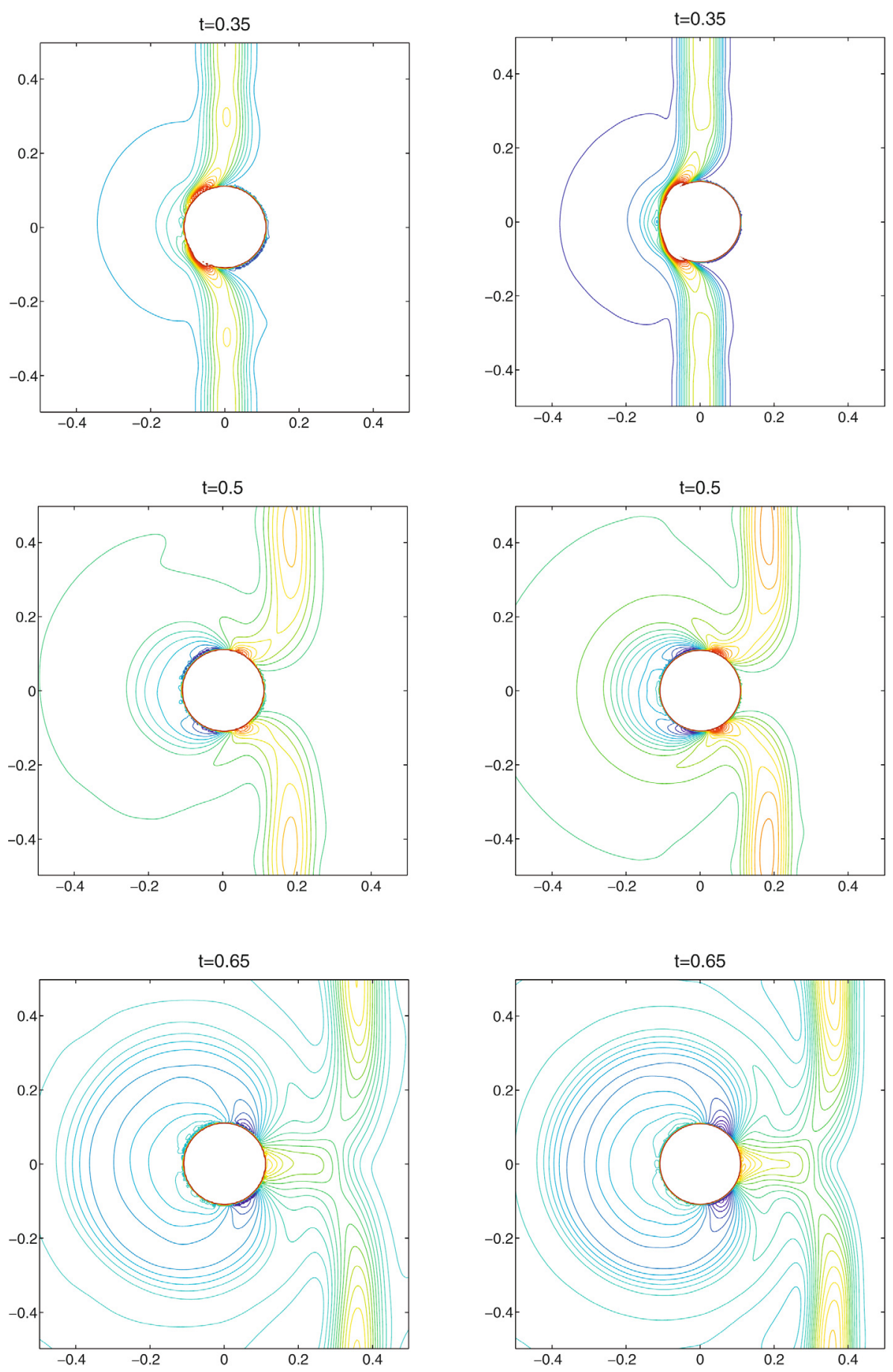

Figure 11. $w$ component of the solution of the IVP (2.1)-(2.4), (3.6), (3.7) on $2 \times 200 \times 200$ (left column) and $2 \times 400 \times 400$ (right column) grids. 

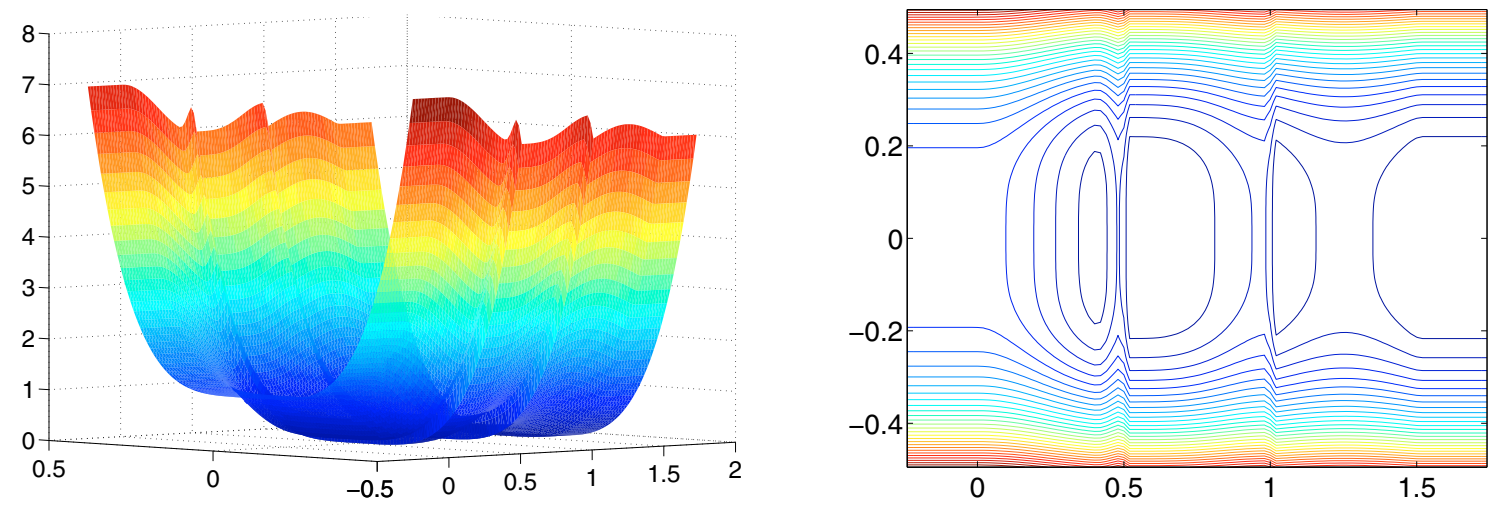

FIGURE 12. River valley topography - three-dimensional view (left) and contour plot (right).

This topography $B$ mimics a mountain river valley, which, together with the surrounding mountains, is shown in Figure 12. We take the following initial data:

$$
w(x, y, 0)=\left\{\begin{array}{ll}
\max \{1.8, B(x, y)\}, & x<0, \\
B(x, y), & x>0,
\end{array} \quad u(x, y, 0) \equiv v(x, y, 0) \equiv 0,\right.
$$

and implement reflecting (solid wall) boundary conditions at all boundaries. These data correspond to the situation when the second of the three dams, initially located at the vertical lines $x=-0.25$ (the left boundary of the computational domain), $x=0$, and $x=1.75$ (the right boundary of the computational domain), breaks down at time $t=0$, the water propagates into the initially dry area $x>0$, and a "lake at rest" steady state is achieved after a certain period of time (this problem is a modification of the 1-D test problem from [21]).

We apply the well-balanced positivity preserving central-upwind scheme to this initial-boundary value problem (IBVP). The friction term in (3.8) is discretized in a straightforward manner. Notice that this affects neither the well-balanced (since $u \equiv v \equiv 0$ at "lake at rest" steady states) nor the positivity preserving (since the first equation has not been changed) properties of our scheme. The solution of the IBVP (3.8)-(3.10) at times $t=0.3,1,4$ and 7 is computed using two different grids. At later times, the computed solution almost does not change, thus the solution at time $t=7$ can be viewed as a numerical "lake at rest" steady state. The solution (the water depth $h$ ) is shown in Figure 13, where one can clearly see the dynamics of the fluid flow as it moves from the region $x<0$ into the initially dry area $x>0$ and gradually settles down into a "lake at rest" steady state. Notice that this state includes dry areas and therefore its computation requires a method that is both well-balanced and positivity preserving on the entire computational domain. To better illustrate the obtained numerical solutions, we plot their 1-D slices along the $y=0$ line in Figures 14, 15, where one can see convergence of the computed solutions to their numerical steady states on two different grids.

\section{Example 4 - Flows in converging-diverging channels}

In the last example, borrowed from [14] (see also [6]), we study water flow in open converging-diverging channel of length 3 with symmetric constrictions of length 1 at the center. The exact geometry of each channel is determined by its breadth, which is equal to $2 y_{\mathrm{b}}(x)$, where

$$
y_{\mathrm{b}}(x)=\left\{\begin{array}{lc}
0.5-0.5(1-d) \cos ^{2}(\pi(x-1.5)), & |x-1.5| \leq 0.5 \\
0.5, & \text { otherwise }
\end{array}\right.
$$



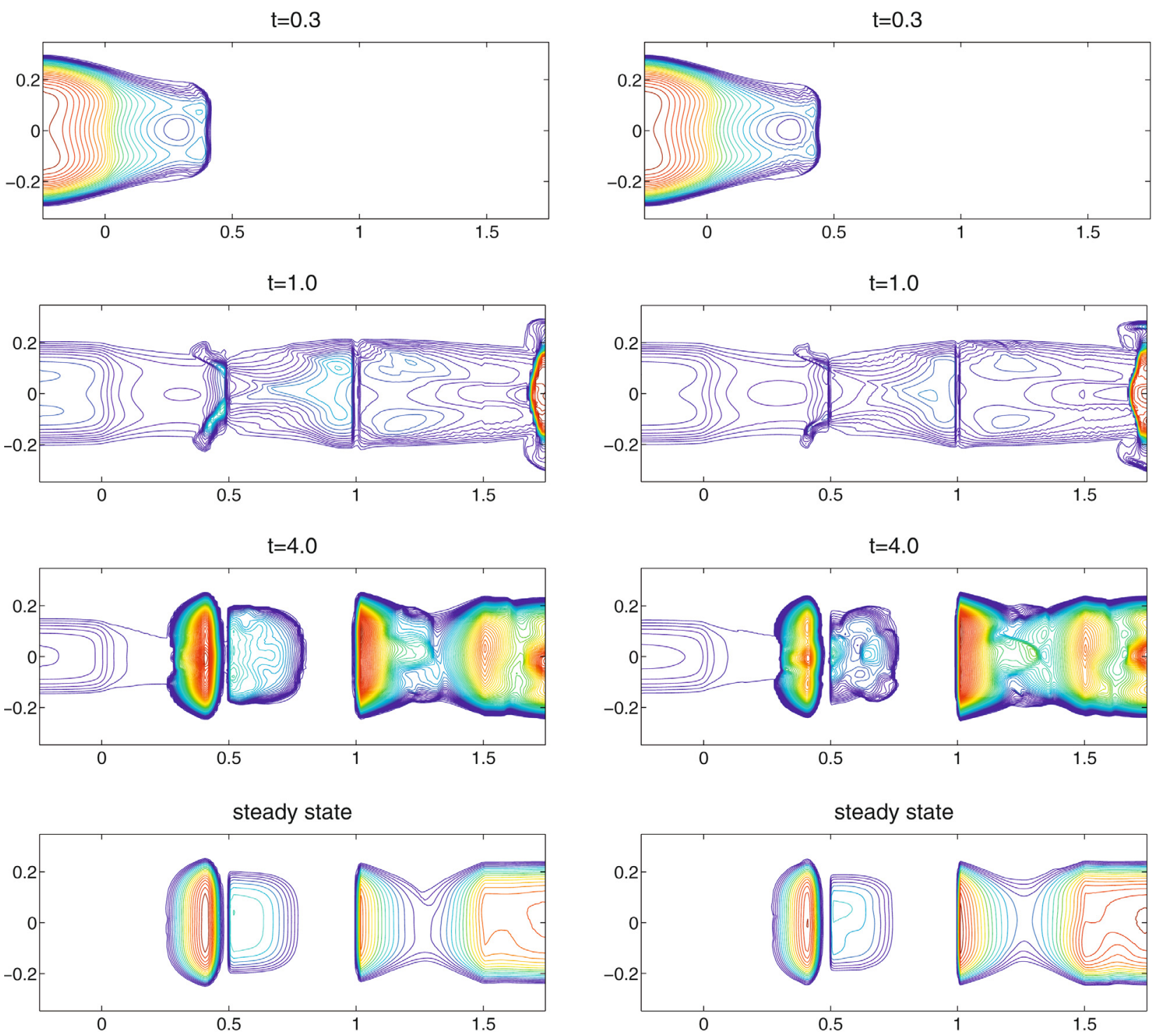

Figure 13. Solution $(h)$ of the IBVP $(2.1)-(2.4),(3.6),(3.7)$ on $2 \times 200 \times 200$ (left column) and $2 \times 400 \times 400$ (right column) grids.

and $d$ is the minimum channel breadth (in our numerical experiments we take $d=0.9$ and $d=0.6$ ). Thus, the computational domain is $[0,3] \times\left[-y_{\mathrm{b}}(x), y_{\mathrm{b}}(x)\right]$, see Figure 16 (left). We take the following initial data:

$$
w(x, y, 0)=\max \{1, B(x, y)\}, \quad u(x, y, 0)=2, \quad v(x, y, 0)=0 .
$$

In the case of a flat bottom $B(x, y) \equiv 0$, these initial data correspond to the data considered in [14] with the Froude number equals to 2. Both the upper and lower $y$-boundaries are reflecting (solid wall), the left $x$-boundary is an inflow boundary with $u=2$ and the right $x$-boundary is a zero-order outflow boundary. 

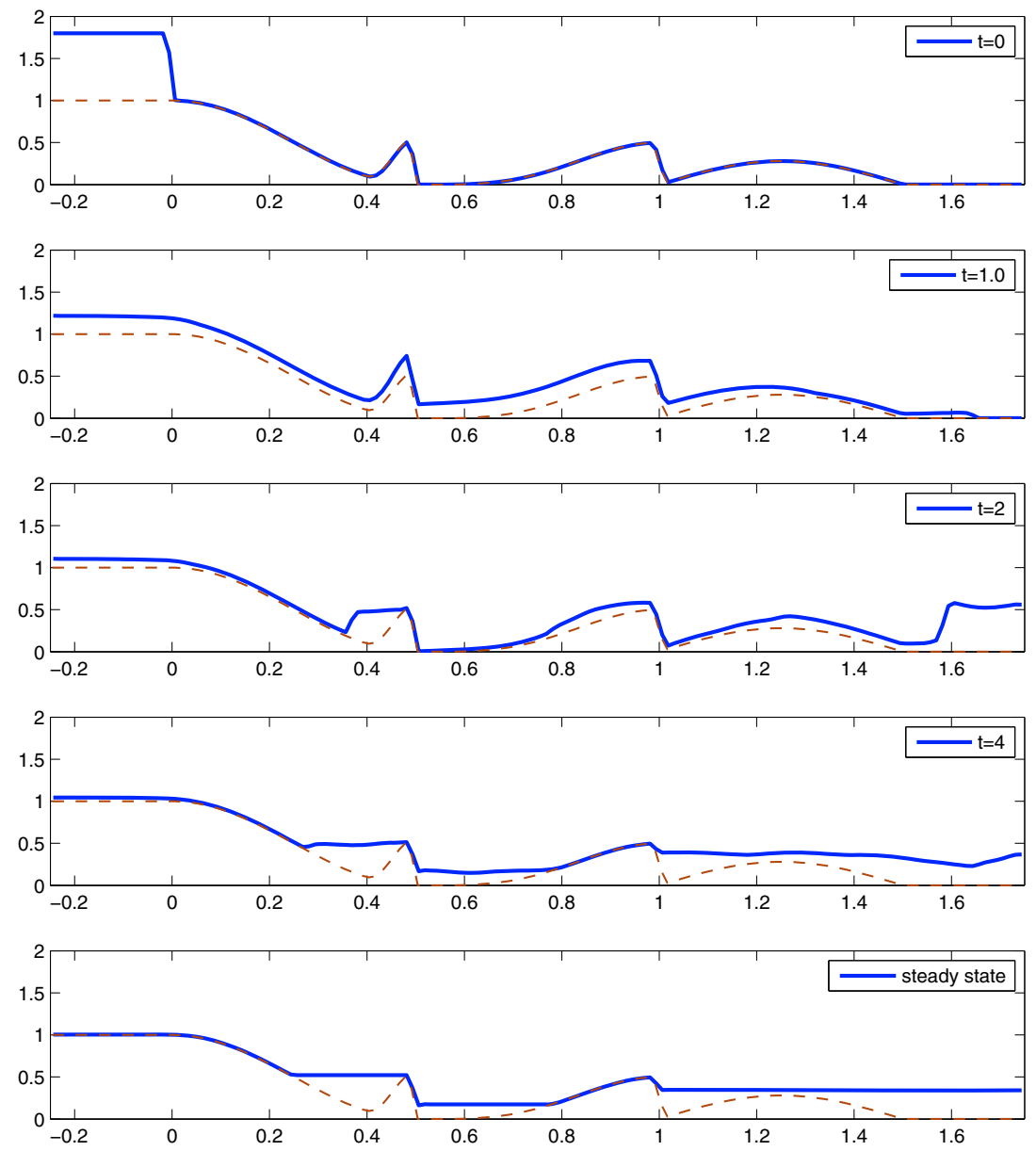

Figure 14. 1-D slice of the water level $w$ (solid line) and the interpolated bottom $B$ (dashed line) from the $2 \times 200 \times 200$ numerical solution shown in Figure 13 .

Finally, the bottom topography, shown in Figure 17, is given by

$$
B(x, y)=B_{\max }\left(\mathrm{e}^{-10(x-1.9)^{2}-50(y-0.2)^{2}}+\mathrm{e}^{-20(x-2.2)^{2}-50(y+0.2)^{2}}\right),
$$

where $B_{\max }$ is a parameter.

We apply the proposed central-upwind scheme on an unstructured triangular mesh obtained from the structured one, outlined in Figure 2, using the mapping

$$
(x, y) \rightarrow\left\{\begin{array}{lc}
\left(x,\left(1-(1-d) \cos ^{2}(\pi(x-1.5))\right) y\right), & |x-1.5| \leq 0.5 \\
(x, y), & \text { otherwise }
\end{array}\right.
$$

The resulting triangulation is shown in Figure 16 (right). We test our method on the following four sets of parameters: $\left(d, B_{\max }\right)=(0.9,0),(0.9,1),(0.9,2)$ and $(0.6,1)$. In every test, we run the simulations on two grids using $2 \times 200 \times 200$ and $2 \times 400 \times 400$ triangles until the steady state (which is, in this case, not a "lake at rest" one) is reached at about $t=2$. The obtained results are shown in Figures 18-21. 

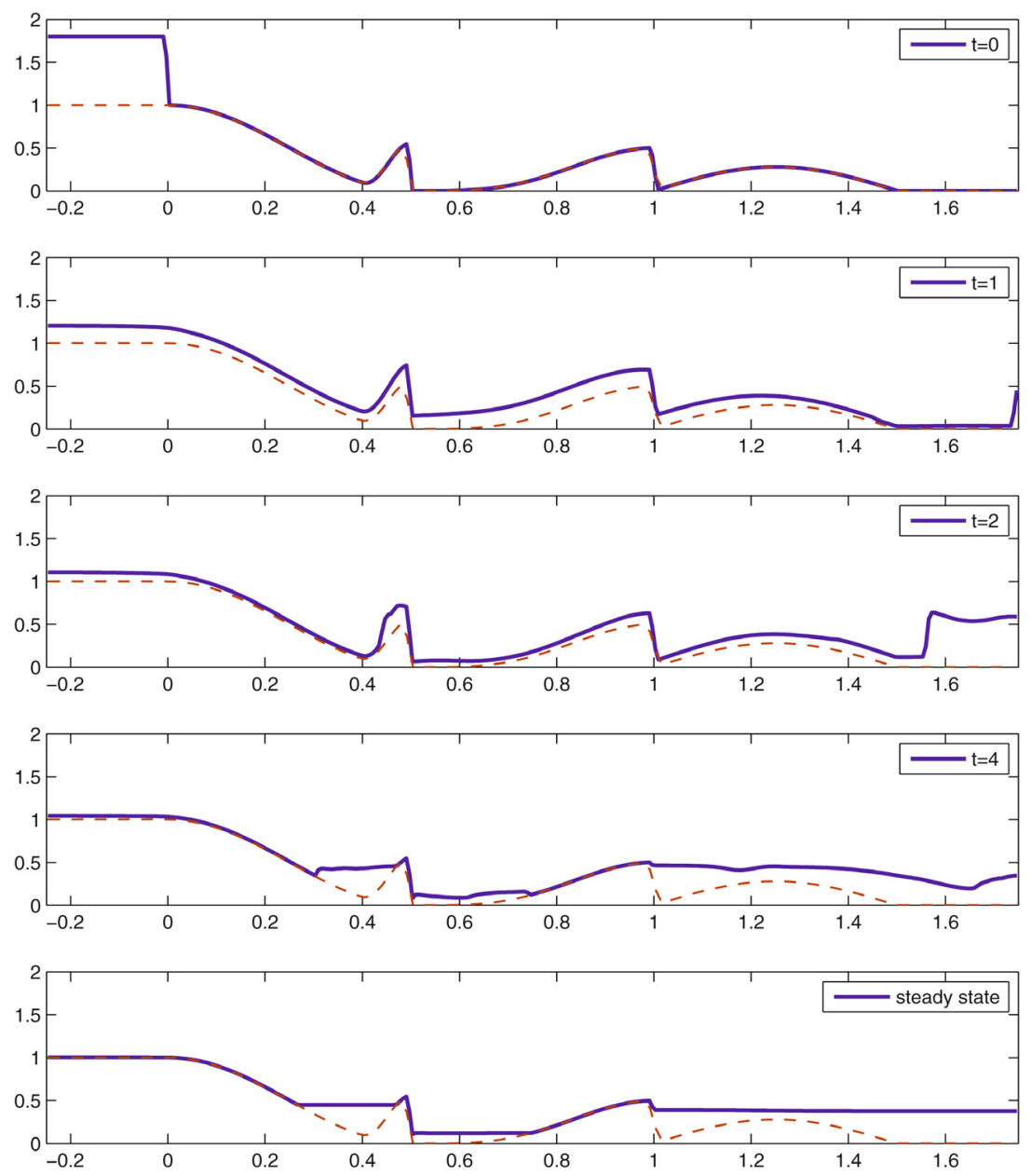

Figure 15. Same as Figure 14, but on the $2 \times 400 \times 400$ grid.
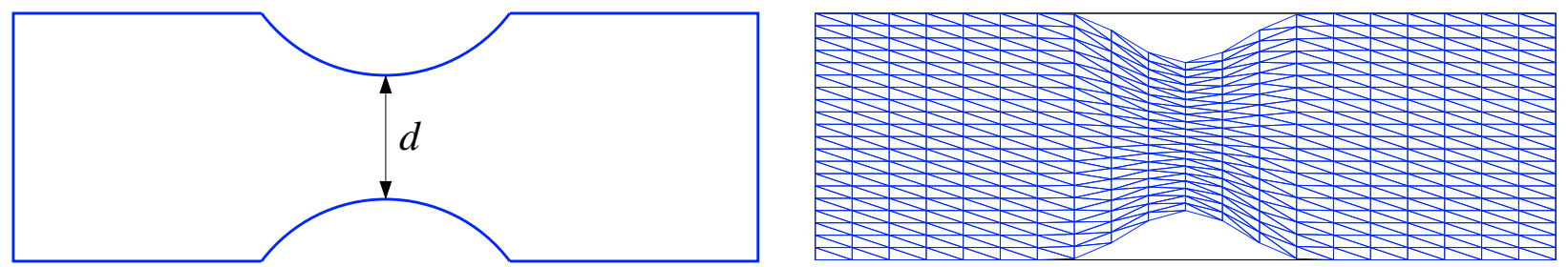

FiguRE 16. Example 4: computational domain (left) and its unstructured triangulation (right).

We first verify that in the flat bottom case, $\left(d, B_{\max }\right)=(0.9,0)$, the results obtained by the central-upwind scheme are in good agreement with the solution computed by an alternative finite-volume scheme, see Figure 18, and compare it with Figure 10(e) in [14].

We then modify the IBVP by including two asymmetric elliptical Gaussian mounds in the bottom topography, that is, by taking $\left(d, B_{\max }\right)=(0.9,1)$. This bottom function is similar to the one used in $[6]$, but in our case 


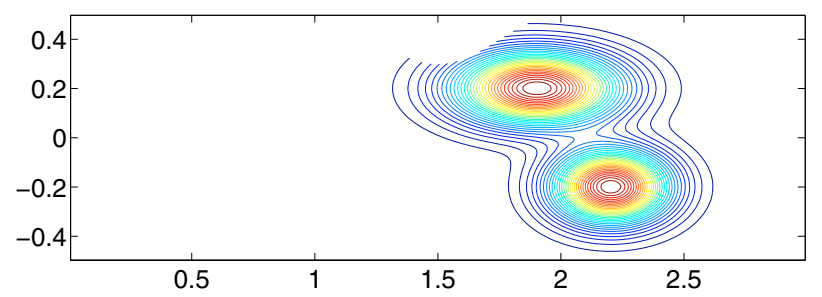

Figure 17. Example 4: bottom topography for $\left(d, B_{\max }\right)=(0.6,1)$.

W

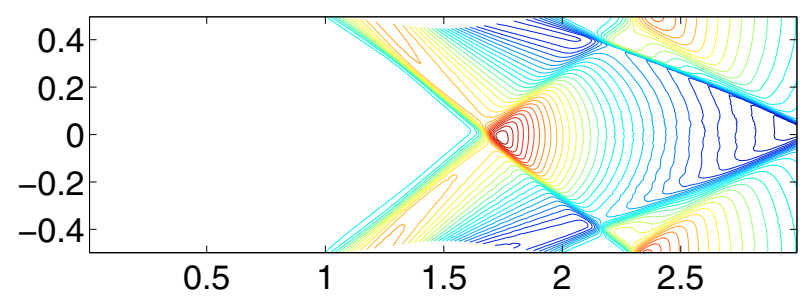

W

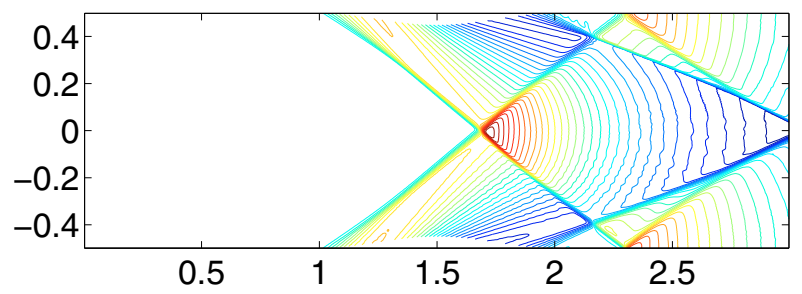

FiguRE 18. Example 4: steady-state solution $(w)$ for $\left(d, B_{\max }\right)=(0.9,0)$ on $2 \times 200 \times 200$ (left) and $2 \times 400 \times 400$ (right) grids.

W

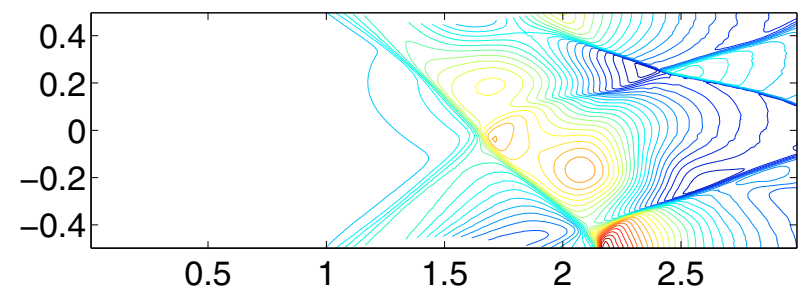

W

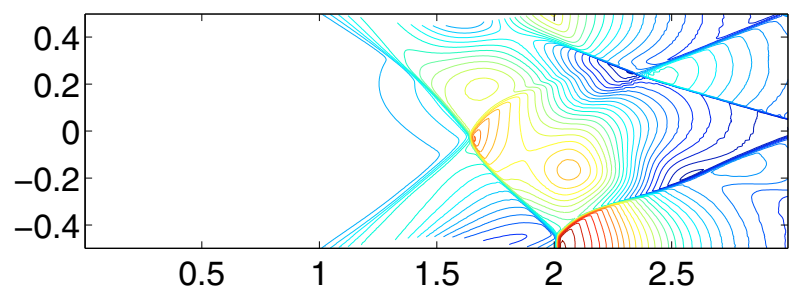

Figure 19. Example 4: steady-state solution $(w)$ for $\left(d, B_{\max }\right)=(0.9,1)$ on $2 \times 200 \times 200$ (left) and $2 \times 400 \times 400$ (right) grids.

the water depth at the top of both mounds is zero. The proposed central-upwind scheme successfully captures the complicated steady-state solution emerging in this case, see Figure 19.

Next, we increase $B_{\max }$ to 2 , which models the presence of two Gaussian-shaped islands. Our scheme still exhibits a superb performance in this case, as one can see in Figure 20, where we show both the water surface $(w)$ and depth $(h)$ to better illustrate the structure of the computed steady-state solution at/near the islands.

Finally, we modify the shape of the channel by taking $d=0.6$, and compute the steady-state solution for $B_{\max }=1$. The results, presented in Figure 21, are of the same high quality as in the case of a wider channel studied above.

Acknowledgements. The research of Y. Epshteyn is based upon work supported by the Center for Nonlinear Analysis (CNA) under the NSF Grant \# DMS-0635983. The research of A. Kurganov was supported in part by the NSF Grant \# DMS-0610430. The research of G. Petrova was supported in part by the NSF Grant \# DMS-0810869 and Award \# KUS-C1-016-04, made by King Abdullah University of Science and Technology (KAUST). 
W
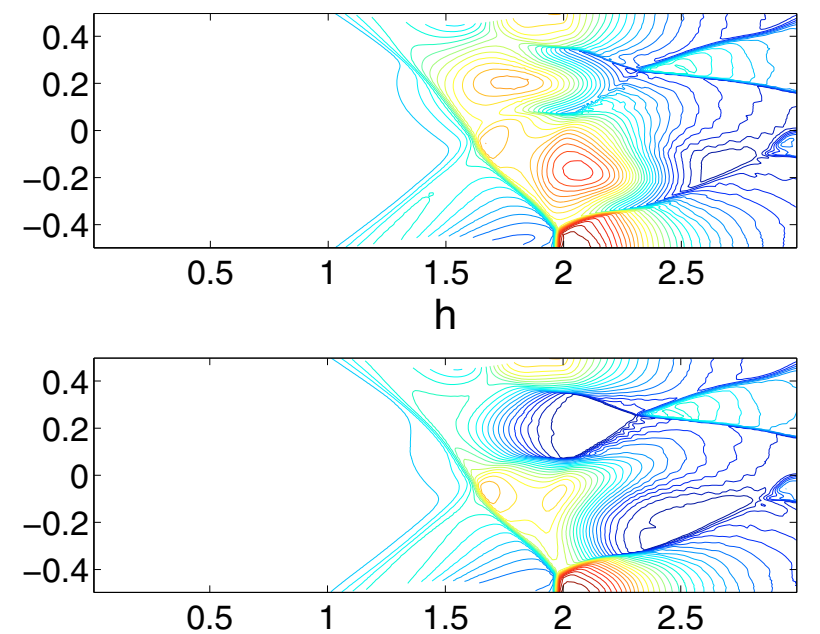

W
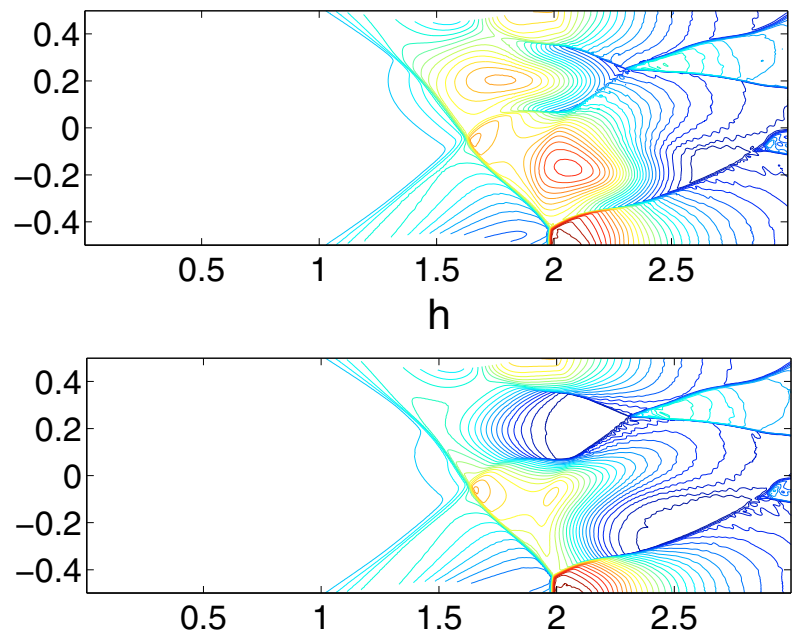

Figure 20. Example 4: steady-state solution $(w-$ top, $h$ - bottom $)$ for $\left(d, B_{\max }\right)=(0.9,2)$ on $2 \times 200 \times 200$ (left) and $2 \times 400 \times 400$ (right) grids.

W

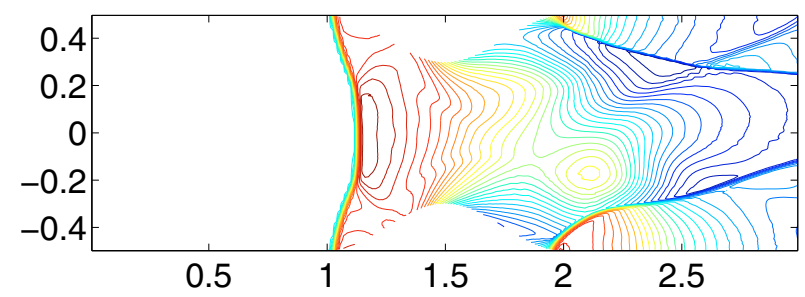

W

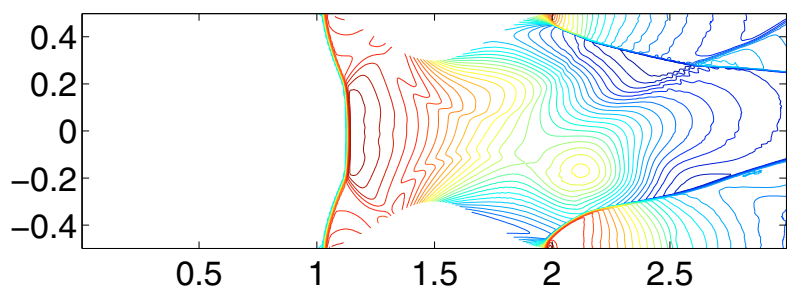

Figure 21. Example 4: steady-state solution $(w)$ for $\left(d, B_{\max }\right)=(0.6,1)$ on $2 \times 200 \times 200$ (left) and $2 \times 400 \times 400$ (right) grids.

\section{REFERENCES}

[1] R. Abgrall, On essentially non-oscillatory schemes on unstructured meshes: analysis and implementation. J. Comput. Phys. 114 (1994) 45-58.

[2] N. Andrianov, Testing numerical schemes for the shallow water equations. Preprint available at http://www-ian.math. uni-magdeburg.de/home/andriano/CONSTRUCT/testing.ps.gz (2004).

[3] P. Arminjon, M.-C. Viallon and A. Madrane, A finite volume extension of the Lax-Friedrichs and Nessyahu-Tadmor schemes for conservation laws on unstructured grids. Int. J. Comput. Fluid Dyn. 9 (1997) 1-22.

[4] E. Audusse, F. Bouchut, M.-O. Bristeau, R. Klein and B. Perthame, A fast and stable well-balanced scheme with hydrostatic reconstruction for shallow water flows. SIAM J. Sci. Comput. 25 (2004) 2050-2065.

[5] F. Bouchut, Nonlinear stability of finite volume methods for hyperbolic conservation laws and well-balanced schemes for sources. Frontiers in Math Series, Birkhäuser Verlag, Basel (2004).

[6] S. Bryson and D. Levy, Balanced central schemes for the shallow water equations on unstructured grids. SIAM J. Sci. Comput. 27 (2005) 532-552.

[7] I. Christov and B. Popov, New nonoscillatory central schemes on unstructured triangulations for hyperbolic systems of conservation laws. J. Comput. Phys. 227 (2008) 5736-5757.

[8] A.J.C. de Saint-Venant, Théorie du mouvement non-permanent des eaux, avec application aux crues des rivière et à l'introduction des marées dans leur lit. C. R. Acad. Sci. Paris 73 (1871) 147-154.

[9] L.J. Durlofsky, B. Engquist and S. Osher, Triangle based adaptive stencils for the solution of hyperbolic conservation laws. J. Comput. Phys. 98 (1992) 64-73. 
[10] T. Gallouët, J.-M. Hérard and N. Seguin, Some approximate Godunov schemes to compute shallow-water equations with topography. Comput. Fluids 32 (2003) 479-513.

[11] J.-F. Gerbeau and B. Perthame, Derivation of viscous Saint-Venant system for laminar shallow water; numerical validation. Discrete Contin. Dyn. Syst. Ser. B 1 (2001) 89-102.

[12] S. Gottlieb, C.-W. Shu and E. Tadmor, High order time discretization methods with the strong stability property. SIAM Rev. 43 (2001) 89-112.

[13] M.E. Hubbard, Multidimensional slope limiters for MUSCL-type finite volume schemes on unstructured grids. J. Comput. Phys. 155 (1999) 54-74.

[14] M.E. Hubbard, On the accuracy of one-dimensional models of steady converging/diverging open channel flows. Int. J. Numer. Methods Fluids 35 (2001) 785-808.

[15] S. Jin, A steady-state capturing method for hyperbolic system with geometrical source terms. ESAIM: M2AN 35 (2001) $631-645$.

[16] S. Jin and X. Wen, Two interface-type numerical methods for computing hyperbolic systems with geometrical source terms having concentrations. SIAM J. Sci. Comput. 26 (2005) 2079-2101.

[17] D. Kröner, Numerical Schemes for Conservation Laws. Wiley, Chichester (1997).

[18] A. Kurganov and D. Levy, Central-upwind schemes for the Saint-Venant system. ESAIM: M2AN 36 (2002) 397-425.

[19] A. Kurganov and C.-T. Lin, On the reduction of numerical dissipation in central-upwind schemes. Commun. Comput. Phys. 2 (2007) 141-163.

[20] A. Kurganov and G. Petrova, Central-upwind schemes on triangular grids for hyperbolic systems of conservation laws. Numer. Methods Partial Diff. Equ. 21 (2005) 536-552.

[21] A. Kurganov and G. Petrova, A second-order well-balanced positivity preserving scheme for the Saint-Venant system. Commun. Math. Sci. 5 (2007) 133-160.

[22] A. Kurganov and E. Tadmor, New high-resolution central schemes for nonlinear conservation laws and convection-diffusion equations. J. Comput. Phys. 160 (2000) 214-282.

[23] A. Kurganov and E. Tadmor, Solution of two-dimensional Riemann problems for gas dynamics without Riemann problem solvers. Numer. Methods Partial Diff. Equ. 18 (2002) 584-608.

[24] A. Kurganov, S. Noelle and G. Petrova, Semi-discrete central-upwind schemes for hyperbolic conservation laws and HamiltonJacobi equations. SIAM J. Sci. Comput. 23 (2001) 707-740.

[25] R.J. LeVeque, Balancing source terms and flux gradients in high-resolution Godunov methods: the quasi-steady wavepropagation algorithm. J. Comput. Phys. 146 (1998) 346-365.

[26] R. LeVeque, Finite volume methods for hyperbolic problems. Cambridge Texts in Applied Mathematics, Cambridge University Press (2002).

[27] K.-A. Lie and S. Noelle, On the artificial compression method for second-order nonoscillatory central difference schemes for systems of conservation laws. SIAM J. Sci. Comput. 24 (2003) 1157-1174.

[28] H. Nessyahu and E. Tadmor, Non-oscillatory central differencing for hyperbolic conservation laws. J. Comput. Phys. 87 (1990) 408-463.

[29] S. Noelle, N. Pankratz, G. Puppo and J. Natvig, Well-balanced finite volume schemes of arbitrary order of accuracy for shallow water flows. J. Comput. Phys. 213 (2006) 474-499.

[30] B. Perthame and C. Simeoni, A kinetic scheme for the Saint-Venant system with a source term. Calcolo 38 (2001) $201-231$.

[31] G. Russo, Central schemes for balance laws, in Hyperbolic problems: theory, numerics, applications II, Internat. Ser. Numer. Math. 141, Birkhäuser, Basel (2001) 821-829.

[32] G. Russo, Central schemes for conservation laws with application to shallow water equations, in Trends and applications of mathematics to mechanics: STAMM 2002, S. Rionero and G. Romano Eds., Springer-Verlag Italia SRL (2005) $225-246$.

[33] P.K. Sweby, High resolution schemes using flux limiters for hyperbolic conservation laws. SIAM J. Numer. Anal. 21 (1984) 995-1011.

[34] B. van Leer, Towards the ultimate conservative difference scheme, V. A second order sequel to Godunov's method. J. Comput. Phys. 32 (1979) 101-136.

[35] M. Wierse, A new theoretically motivated higher order upwind scheme on unstructured grids of simplices. Adv. Comput. Math. 7 (1997) 303-335.

[36] Y. Xing and C.-W. Shu, High order finite difference WENO schemes with the exact conservation property for the shallow water equations. J. Comput. Phys. 208 (2005) 206-227.

[37] Y. Xing and C.-W. Shu, A new approach of high order well-balanced finite volume WENO schemes and discontinuous Galerkin methods for a class of hyperbolic systems with source terms. Commun. Comput. Phys. 1 (2006) 100-134. 